\title{
New conformal gauging and the electromagnetic theory of Weyl
}

\author{
James T. Wheeler ${ }^{\mathrm{a})}$ \\ Department of Physics, Utah State University, Logan, Utah 84322
}

(Received 7 July 1997; accepted for publication 3 September 1997)

\begin{abstract}
A new eight-dimensional conformal gauging solves the auxiliary field problem and eliminates unphysical size change from Weyl's electromagnetic theory. We derive the Maurer-Cartan structure equations and find the zero curvature solutions for the conformal connection. By showing that every one-particle Hamiltonian generates the structure equations we establish a correspondence between phase space and the eight-dimensional base space, and between the action and the integral of the Weyl vector. Applying the correspondence to generic flat solutions yields the Lorentz force law, the form and gauge dependence of the electromagnetic vector potential and minimal coupling. The dynamics found for these flat solutions applies locally in generic spaces. We then provide necessary and sufficient curvature constraints for general curved eight-dimensional geometries to be in 1-1 correspondence with four-dimensional Einstein-Maxwell space-times, based on a vector space isomorphism between the extra four dimensions and the Riemannian tangent space. Despite part of the Weyl vector serving as the electromagnetic vector potential, the entire class of geometries has vanishing dilation, thereby providing a consistent unified geometric theory of gravitation and electromagnetism. In concluding, we give a concise discussion of observability of the extra dimensions. (C) 1998 American Institute of Physics. [S0022-2488(98)02601-2]
\end{abstract}

\section{INTRODUCTION}

In 1918, following immediately on the heels of Einstein's introduction of general relativity, Weyl proposed a generalization of Riemanian space-time structure ${ }^{1}$ to allow for local changes in the standard of length. When the gauge field required to insure this symmetry has nonvanishing curl, the lengths of vectors change as they undergo parallel transport. This nicely completes the picture of Riemann in which vectors are rotated but not changed in length. In Weyl geometry, all properties of transported vectors-magnitude and direction-are subject to the transport law.

Weyl identified the gauge field of local dilations with the electromagnetic potential, thereby explaining the gauge freedom of that potential and giving a geometric interpretation of electromagnetic forces. Unfortunately, the theory is inconsistent with experience because it predicts changes in the sizes of objects depending on their paths. Applied, for example, to the case of atoms moving in a background electromagnetic field, the predicted size changes would produce substantial unobserved broadening of atomic spectral lines.

The simplicity and naturalness of Weyl geometry together with the promise of a geometric understanding of electromagnetism makes the failure of Weyl's physical theory puzzling. Indeed, Weyl ${ }^{1-3}$ and many other physicists ${ }^{4-22}$ included proposed alternative versions of the physical interpretation in attempts to make the theory consistent. Ultimately, the work led to the current $\mathrm{U}(1)$ gauge theory of electromagnetism, which maintains much of the beauty of the original proposal without inconsistent predictions such as changes in atomic spectra.

In the present work, we show that a new conformal gauging avoids the standard objection ${ }^{5}$ to this 80-year-old theory of Weyl. Since the conformal group contains the Weyl group, the spaces

${ }^{a)}$ Electronic-mail: jwheeler@cc.usu.edu 
resulting from its gauging share certain properties with Weyl's original gauge theory of electromagnetism. However, the eight-dimensional character of the new gauging and its interpretation as a generalization of phase space eliminate the problem of unphysical size change. Before discussing these new conformal spaces, which we call biconformal geometries, we review some results from previous studies of the conformal group.

The conformal group is the most general set of transformations preserving ratios of infinitesimal lengths. On four-dimensional space-time the conformal group is 15 dimensional, including Lorentz transformations, translations, inverse translations, and dilations. The Lorentz transformations and translations are well known, while the dilations are simple rescalings. The inverse translations have alternatively been called special conformal transformations, conformal boosts, accelerations, or elations. We choose the term inverse translations because these transformations are pure translations at infinity. This is easily seen by translating the inverse coordinate $y^{\mu}$ $=-x^{\mu} / x^{2} \rightarrow y^{\mu}+a^{\mu}$ and then inverting again.

We will also make use of several subgroups of the conformal group. The Lorentz transformations and translations together form the Poincare group, while the Lorentz transformations and the dilations form the homogeneous Weyl group. Adding the translations to the Weyl group gives the inhomogeneous Weyl group. The Lorentz transformations, dilations, and inverse translations also form an inhomogeneous Weyl group with the only difference being that the translations and inverse translations have opposite behavior under dilations.

Considerable attention ${ }^{23-27}$ has been given to four-dimensional space-times which result from gauging the conformal group. In addition to the usual metric and local Lorentz structures of space-time, these models are expected, a a priori, to possess five gauge vectors-one for dilations and four for the inverse translations. However, it was long thought that the inverse translations could not be gauged, because the corresponding current is position dependent. From a slightly different perspective, Mansouri et al. ${ }^{23,24}$ argue that making the inverse translations local is redundant with general coordinate transformations so that the corresponding gauge fields have no new effect. Nonetheless, Crispim Romão, Ferber, and Freund, ${ }^{25,26}$ and independently Kaku, Townsend, and Van Nieuwenhuizen ${ }^{27}$ show how to gauge the inverse translations, but find them algebraically removable from the problem. This problem is in fact generic: in any fourdimensional scale-invariant torsion-free field theory with action built quadratically from the curvatures of the conformal group these four inverse translational gauge fields may be algebraically removed from the problem. ${ }^{28}$ The local inhomogeneous Weyl symmetry of such theories always has an effective field theory equivalent to one based on the homogeneous Weyl group, and therefore sheds no light on Weyl's theory.

By contrast, consideration of the fixed points of the various conformal transformations suggests the use of an eight-dimensional base space instead of a four-dimensional base space, corresponding to the existence of eight distinct translational generators in the Lie algebra. Requiring an eight-dimensional base space makes the gauge fields of the inverse translations act as part of the solder form on the base space, so these fields necessarily remain independent. Even in the case of a fully flat biconformal space this solder form contains nontrivial physical information.

Our present investigation of this new conformal gauging focuses principally on the study of flat biconformal spaces, because this is where the connection with well-known systems should be most evident and because the dynamics of flat spaces will also apply locally in generic spaces. Unlike Riemannian geometry, there is an entire nontrivial class of flat solutions. We first derive this class of solutions. Next, we show that the flat spaces possess a symplectic structure which allows their interpretation as one-particle phase spaces and provides a concise description of the corresponding Hamiltonian dynamics. Applying this Hamiltonian correspondence to the most general flat solution gives a geometric derivation of the Lorentz force law, in strong contrast to the original Weyl theory. After indicating briefly how the biconformal electromagnetic theory connects satisfactorily to U(1) gauge theory, we show how to introduce Einstein gravitation, arbitrary additional fields, and electromagnetic sources into the biconformal structure. 
The layout of the paper is as follows. In Sec. II we develop biconformal structure as a conformal fiber bundle. We give particular attention to the general procedure for building connections on manifolds based on an underlying symmetry group ${ }^{29-32}$ because it is this procedure which motivates our use of an eight-dimensional base manifold. The construction consists of two steps. First, an elementary geometry is found by taking the quotient of a given symmetry group by a subgroup containing no normal subgroup of the full group. Then, the connection of the resulting principal fiber bundle is generalized by including curvature. As an example we review the construction of general space-times by gauging the Poincare group. ${ }^{33-36}$ We then implement these techniques to gauge the conformal group in a new way. First, we construct an elementary geometry as the quotient, $\mathscr{C} / \mathscr{C}_{0}$, of the conformal group, $\mathscr{C}$, by its isotropy subgroup, $\mathscr{C}_{0}$. This gives a conformal Cartan connection on an eight-dimensional manifold. Next, we generalize the connection to that of a curved eight-dimensional manifold with the seven-dimensional homogeneous Weyl group as fiber by the addition of horizontal curvature two-forms to the group structure equations. The resulting eight-dimensional base manifold is called a biconformal space and the full fiber bundle is called the biconformal bundle.

Our first principal result is presented in Sec. III where we derive the flat solution for a frame field satisfying the zero-curvature Maurer-Cartan structure equations of biconformal space.

In Secs. IV and V we establish our next claim: the classical Hamiltonian dynamics of a single-point particle is equivalent to the specification of a seven-dimensional surface in flat biconformal space. As a consequence of the form of the structure equations, there necessarily exists a set of preferred curves satisfying the Hamiltonian equations of motion. This claim is established in two steps. First, in Sec. IV, we show that the classical Hamiltonian description of a point particle defines a class of eight-dimensional differential geometries with structure equations of manifestly biconformal type. Then in Sec. V we show that there is an embedding, unique up to local symplectic changes of basis, of the Hamiltonian system into a flat biconformal geometry. We also demonstrate the necessary existence of a preferred set of curves in the biconformal space satisfying the Hamiltonian equations of motion. The unique local equivalence between hypersurfaces in flat biconformal geometry and Hamiltonian systems provides a clear physical interpretation of the geometric variables of biconformal space.

The central importance of this second result is in definitively establishing the physical interpretation of the new conformal gauging. Our main goal is not to provide an alternate formulation of classical mechanics, but rather to use this embedding as a guide to subsequent physical interpretation of the elementary biconformal variables. Given the result of Sec. IV, that a classical single-particle Hamiltonian system generates a class of biconformal space-times, and its converse in Sec. V, we can conclude that for an isolated test particle in a general biconformal space the four new coordinates may be interpreted locally as the corresponding generalized particle momentum. Our identification therefore provides a physical correspondence principle for biconformal spaces. While in general spaces the extra dimensions will not necessarily represent momentum globally, we can always take the limit of a tightly confined field in a local Lorentz frame, for which the extra dimensions will permit the momentum interpretation. Indeed, when nonflat biconformal spaces are investigated in Sec. VII, we will see that the "momentumlike", co-solder form contains the stress-energy tensor of any gravitational sources. This generalization from momentum to the stress-energy tensor is exactly what one would anticipate in moving from a particle interpretation to a field interpretation.

For our third result, in Sec. VI, we apply the Hamiltonian correspondence of the previous sections to the general solution for flat biconformal space, to show how that flat solution predicts the following properties of the electromagnetic vector potential:

(1) The four-vector form of the potential. This is nontrivial, since it involves the reduction of the eight-dimensional one-form $\omega_{0}^{0}=\omega_{0 \mu}^{0}(x, y) \mathbf{d} x^{\mu}+\omega_{0}^{0 \mu}(x, y) \mathbf{d} y_{\mu}$ to a four-dimensional oneform, $\alpha_{\mu}(x) \mathbf{d} x^{\mu}$ on space-time.

(2) Its usual gauge dependence $\alpha^{\prime}(x)=\alpha(x)+\mathbf{d} \phi$. 
(3) Minimal coupling $p_{a} \rightarrow p_{a}-\lambda \alpha_{a}$.

(4) The correct equation of motion for a charged particle moving under its influence.

This section therefore provides a consistent realization of Weyl's goal ${ }^{l}$ of expressing electrodynamics in terms of the dilational gauge field. Upon moving to more general biconformal spaces, the remarks of the preceding paragraph imply that in a local Lorentz frame an isolated, charged test particle will move according to the Lorentz force law.

These predictions of electromagnetic effects in biconformal space follow directly from the relationship of biconformal space to phase space and Hamiltonian dynamics, developed in Secs. IV and V. No further assumption is necessary to derive the Lorentz force law. At the same time, the biconformal theory predicts constancy of size. There is an entire class of flat biconformal spaces which includes sufficient freedom in the gauge fields to account for the classical electrodynamics of a charged point particle in an arbitrarily specified background electromagnetic field. The flatness condition includes vanishing dilational curvature, so none of these geometries leads to size change of any kind. In sharp contrast to Weyl geometry in which vanishing dilation implies vanishing gauge vector, the dilational gauge vector in flat biconformal space is required to be nonzero.

Section VI ends with a brief comparison of the biconformal model with Weyl's original gauge theory and with the standard U(1) model of electromagnetism.

In Sec. VII, we show how to introduce arbitrary gravitational and electromagnetic sources so that the results of Sec. VI remain valid in the resulting class of curved biconformal spaces. The entire class has vanishing dilation, and the electromagnetic and gravitational fields satisfy the Maxwell and Einstein equations, respectively. This section therefore provides a consistent realization of Weyl's goal of a unified geometric theory of gravitation and electromagnetism. While other fundamental interactions have been discovered since Weyl's time, the inclusion of both of the large scale forces in a geometric theory must be considered a step in the right direction; moreover, biconformal spaces contain additional fields which are not studied here. These additional fields might have an interpretation as the additional interactions. In any case, there is always the possibility of adding internal symmetries beyond the conformal symmetry.

The final section consists of a detailed discussion of possible consequences of regarding energy and momentum variables as four of the coordinates in an eight-dimensional space. We consider the necessary isomorphism of mathematical structure, transformation properties, and dynamical laws that must hold between momentum space variables and the biconformal co-space variables. Collisions and interactions are discussed with attention given to continuity and the proper proximity of colliding particles. Finally, we point to some experimental results which suggest a coordinatelike behavior of momentum variables.

\section{THE CONSTRUCTION OF SPACE-TIMES WITH LOCAL SYMMETRIES}

Before defining and interpreting biconformal spaces at the end of this section, we require some background motivating their construction. While it is customary to begin the discussion of a differential geometry with the specification of a manifold and metric pair, $(\mathscr{l}, g)$, our present interest lies in developing a space-time model beginning with locally determined symmetry considerations. This allows us to construct geometries which a priori possess specified local symmetries. It is in part the elements of this construction which motivate our choice of an eight-dimensional base manifold for conformal gauging instead of the usual four-dimensional picture. This in turn requires us to interpret the extra four dimensions. The interpretation of the extra coordinates in the zero curvature limit is accomplished in Secs. IV-VI.

We consider symmetry groups which include the local Lorentz symmetry of space-time, seeking a procedure for developing a connection on a space-time $(\mathscr{L}, g)$ from a knowledge of an experimentally determined (i.e., "global") symmetry group. We use the well-known techniques of Cartan and Klein, ${ }^{29-32,37-39}$ proceeding as follows. We begin with a Lie group, $\mathscr{G}$, and a 
subgroup $\mathscr{G}_{0}$ called the isotropy subgroup. The isotropy subgroup should contain no normal subgroup of $\mathscr{G}$ if the full group is to act effectively and transitively on the base space. Defining a projection onto the base space as the quotient $\mathscr{G} / \mathscr{G}_{0}$, the group manifold becomes a fiber bundle, called by Klein an elementary geometry. The full group will act effectively and transitively on the base space of the elementary geometry. The base space will be a manifold if and only if the fibration is regular, i.e., there exists a neighborhood of each point of any fiber which that fiber intersects only once. This regularity condition holds for the groups we consider. This base space of the elementary geometry therefore provides a manifold upon which we now generalize the connection to a Cartan connection by introducing curvature.

The generalization to a Cartan connection occurs by only requiring the action of the full group to be detectable on curves instead of globally. Specifically, we may introduce into the structure equations any curvature two-forms consistent with the resulting Bianchi identities and the following requirement. Let $\mathscr{P}(\lambda)$ be any curve in the bundle, let $\omega_{j}^{i}$ be a connection on the bundle, and let $f: \mathscr{G} \rightarrow V^{N^{2}}$ be a linear representation of $\mathscr{G}$ by $N \times N$ matrices. Then integrating

$$
\mathbf{d} f_{j}^{i}=\omega_{j}^{k} f_{k}^{i}
$$

along $\mathscr{P}(\lambda)$ yields a group transformation $f_{j}^{i}\left(\lambda_{0}\right)$ at each point $\mathscr{P}\left(\lambda_{0}\right)$. The connection $\omega_{j}^{i}$ will be a Cartan connection if the transformation $f_{j}^{l}(\lambda)$ depends only on the projection of the curve $\mathscr{P}(\lambda)$ into the base space. This condition holds if and only if the curvatures are horizontal, i.e., bilinear in those connection forms which vanish on the fibers. The generalization to a curved connection occurs because different curves in the base space between the same pair of points can give different group elements.

If the connection is linear, we can express this condition in terms of mappings of orthonormal frames. Consider again a curve $\mathscr{P}(\lambda)$, an initial point $\mathscr{P}(0)$ on the curve, and a frame $\mathscr{E}_{a}(0)$ at the point. We then can demand that there shall exist a transformation from the full group $\mathscr{G}$ mapping the initial pair $\left(\mathscr{P}(0), \mathscr{E}_{a}(0)\right)$ to a corresponding pair $\left(\mathscr{P}(\lambda), \mathscr{E}_{a}(\lambda)\right)$ for any point $\mathscr{P}(\lambda)$ on the curve.

\section{A. Gauging the Poincaré group}

We illustrate the method using the Poincaré group. The group may be described locally by its structure equations (equivalent to its Lie algebra), which take the form

$$
\mathbf{d} \omega_{b}^{a}=\omega_{b}^{c} \wedge \omega_{c}^{a}, \quad \mathbf{d} \omega^{a}=\omega^{b} \wedge \omega_{b}^{a},
$$

where the one-forms $\omega_{b}^{a}, \omega^{a}(a, b=1,2,3,4)$ span the ten-dimensional group manifold. The only subgroup $\mathscr{G}_{0}$ containing no normal subgroup of $\mathscr{G}$ but which does include the Lorentz group is the Lorentz group itself, since the translations form a normal subgroup. The quotient $\mathscr{G} / \mathscr{G}_{0}$ leads to a four-dimensional base space with Lorentz fibers. It is easy to see that the base space is Minkowski space, and therefore the elementary geometry is the bundle of orthonormal frames over Minkowski space-time.

Continuing with the Lorentz bundle, we alter the connection to that of a curved base space by adding curvature two-forms, $\Omega_{b}^{a}$ and $\Omega^{a}$, to the structure equations. Clearly there is one curvature two-form for each generator of the original Lie group. Furthermore, each curvature component is horizontal, depending only on the forms $\omega^{a}$ which vanish on the fibers. Functionally, the curvature components depend only on the quotient space, $\mathscr{G} / \mathscr{G}_{0}$. Thus,

$$
\mathbf{d} \omega_{b}^{a}=\omega_{b}^{c} \wedge \omega_{c}^{a}+\Omega_{b}^{a}, \quad \mathbf{d} \omega^{a}=\omega^{b} \wedge \omega_{b}^{a}+\Omega^{a},
$$

or, in more familiar notation, 


$$
\mathbf{R}^{a}{ }_{b}=\mathbf{d} \omega^{a}{ }_{b}+\omega^{a}{ }_{c} \wedge \omega^{c}{ }_{b} \quad T^{a}=\mathbf{d e}^{a}+\omega^{a}{ }_{b} \wedge \mathbf{e}^{b},
$$

where $\omega^{a}{ }_{b}$ is the spin connection, $\mathbf{e}^{a}=\omega^{a}$ is the vierbein, $\mathbf{R}_{b}^{a}$ is the curvature two-form, and $\mathbf{T}^{a}$ is the torsion. Notice that we will always write the metric explicitly, so we need not offset upper and lower indices. The horizontality condition, that $\mathbf{R}_{b}^{a}=\mathbf{R}_{b c d}^{a} \mathbf{e}^{c} \wedge \mathbf{e}^{d}$ with no terms such as $\mathbf{R}_{b c d}^{a}{ }^{e} \mathbf{e}^{c} \wedge \omega_{e}^{d}$ or $\mathbf{R}^{a}{ }_{b c d}{ }^{e f} \omega_{e}^{c} \wedge \omega_{f}^{d}$, guarantees that the generator of a Poincare transformation found by integrating the connection one-forms along any curve $\mathscr{P}(\lambda)$ will depend only on the projection of the curve into the base manifold, $\pi(\mathscr{P}(\lambda))$. Specifically, define

$$
\mathbf{d}_{1} \mathscr{P}=\omega^{a} \mathscr{E}_{a}, \quad \mathbf{d}_{1} \mathscr{E}_{a}=\omega_{a}^{b} \mathscr{E}_{b},
$$

as the change in the point $\mathscr{P}$ and the vector frame $\mathscr{E}_{b}$ along any curve in the bundle, where $\mathbf{d}_{1}$ is the one-dimensional exterior derivative on the curve. It is convenient to introduce a full tendimensional frame $\left(\mathscr{E}_{a}, \mathscr{F}_{a}^{b}\right)$ at the initial point, with the frame $\mathscr{E}_{a}$ being the horizontal part. We choose $\left(\mathscr{E}_{a}, \mathscr{F}_{a}^{b}\right)$ to be dual to $\left(\omega^{a}, \omega_{b}^{a}\right)$ :

$$
\begin{gathered}
\omega^{a}\left(\mathscr{E}_{b}\right)=\delta_{b}^{a}, \quad \omega^{a}\left(\mathscr{F}_{c}^{b}\right)=0, \\
\omega_{b}^{a}\left(\mathscr{E}_{c}\right)=0, \quad \omega_{b}^{a}\left(\mathscr{F}_{d}^{c}\right)=\delta_{d}^{a} \delta_{b}^{c} .
\end{gathered}
$$

When Eqs. (2.3) are integrated along a curve $\mathscr{P}(\lambda)$ we find a new point-frame pair at each value of $\lambda$. Now let $\mathscr{P}(\lambda)$ be the closed perimeter of an arbitrary infinitesimal plaquette with area element $S$. Then explicit evaluation of the integral of $\mathbf{d}_{1} \mathscr{P}$ around the plaquette gives

$$
\oint_{\mathscr{P}} \mathbf{d}_{1} \mathscr{P} \approx \Omega^{a}(S) \mathscr{E}_{a}(0)
$$

A general infinitesimal surface element $S$ may be expanded in terms of the full frame at the initial point as

$$
S=S^{a b} \mathscr{E}_{a} \wedge \mathscr{E}_{b}+S_{c}^{a b} \mathscr{F}_{a}^{c} \wedge \mathscr{E}_{b}+S_{c d}^{a b} \mathscr{F}_{a}^{c} \wedge \mathscr{F}_{b}^{d}
$$

Now taking $\Omega^{a}$ horizontal, $\Omega^{a}=\Omega_{b c}^{a} \omega^{b} \wedge \omega^{c}$, we use Eqs. (2.4) to evaluate

$$
\begin{aligned}
\Omega^{a}(S) & =\Omega_{b c}^{a}\left[S^{d e} \omega^{b}\left(\mathscr{E}_{d}\right) \omega^{c}\left(\mathscr{E}_{e}\right)+S_{f}^{d e} \omega^{b}\left(\mathscr{F}_{d}^{f}\right) \omega^{c}\left(\mathscr{E}_{e}\right)+S_{f g}^{d e} \omega^{b}\left(\mathscr{F}_{d}^{f}\right) \omega^{c}\left(\mathscr{F}_{e}^{g}\right)\right] \\
& =\Omega_{b c}^{a} S^{d e} \delta_{d}^{b} \delta_{e}^{c}=\Omega^{a}(\pi(S)),
\end{aligned}
$$

so that the path dependence of the point $\mathscr{P}(\lambda)$ depends only on the projection of the loop into the base manifold. A completely analogous argument holds for the integral of $\mathscr{E}_{a}$ and the horizontality of $\Omega_{b}^{a}$.

Equations (2.2) or $\left(2.2^{\prime}\right)$ now describe a curved four-dimensional space-time with local Lorentz symmetry. The second curvature, $\Omega^{a}=\mathbf{T}^{a}$, allows the inclusion of torsion. Because the remaining Lorentz symmetry of the fibers does not mix the components of the torsion with the components of the Riemann curvature, the usual specification of general relativity, $\Omega^{a}=0$, is consistent. General relativity also requires identification of the cotangent space $T^{*}$ with the space spanned by the solder form $\mathbf{e}^{a}$. This identification is automatic here because $\mathbf{e}^{a}$ is required a priori to span the base space $\mathscr{G} / \mathscr{G}_{0}$. As we have carried out the construction here, the topology of the manifold is that of the quotient, $R^{4}$, but nontrivial base spaces are easily achieved by specifying 
an arbitrary manifold $\mathscr{L} b$ having the same (Minkowski) cotangent space and structure equations, (2.2). This substitution of $\mathscr{L}$ for $\mathscr{G} / \mathscr{G}_{0}$ is allowed because it alters only topological properties, while all of the structures of interest described above are local.

\section{B. Biconformal gauging}

Understanding a symmetry-based approach has significant advantages as we now turn to our examination of the conformal group. The essential point is that, in contrast to the Poincare example, the presence of inverse translations means that there are no normal subgroups, making the choice of isotropy subgroup $\mathscr{C}_{0}$ nontrivial. While biconformal space is based on the choice of the homogeneous Weyl group as $\mathscr{C}_{0}$, it is instructive to first consider what happens if we use the inhomogeneous Weyl group instead.

As described in the Introduction, considerable attention ${ }^{23-27}$ has been given to models which result from the choice of the 11-dimensional inhomogeneous Weyl group as $\mathscr{C}_{0}$. This choice is natural enough since it gives the same base manifold as in the Poincare case. It would appear that this model simply extends the Lorentz fiber symmetry of the local Poincare model to include dilations and inverse translations. However, in generic field theories based on this bundle the four components of the connection corresponding to the inverse translations are generically auxiliary and may be algebraically removed from the problem. ${ }^{28}$ The resulting field theory, equivalent to one based on the homogeneous Weyl group, has had at best mixed success as a field theory. In any case, no new symmetries or additional physical fields have been gained in passing from the Weyl group to the full conformal group. By contrast, choosing $\mathscr{C}_{0}$ to be the homogeneous Weyl group from the start, we retain the full 15 degrees of freedom of the original group and acquire many new fields, with the simplest example of a biconformal space having a natural physical interpretation in terms of Hamiltonian dynamics.

We arrive at the biconformal model if we ask what property of the Lorentz transformations made them a suitable fiber symmetry for the Poincare group. The requirement that the isotropy subgroup should have no subgroup normal in the Poincare group ruled out the translations, which form a normal subgroup of the Poincare group. The Lorentz symmetry is therefore the only possible fiber symmetry. However, there is another way to see that we should use the Lorentz group as the isotropy subgroup, based on fixed points. On Minkowski space, the class of translations has no fixed points, while the class of Lorentz transformations leaves the origin fixed (hence the name, "isotropy subgroup"). Thus, we can distinguish the isotropy subgroup of the fibering from the "translational" symmetry of the base manifold by counting fixed points.

Returning to the conformal group, we find that when we distinguish the conformal transformations based on their fixed points, there are simply eight translations acting on compactified Minkowski space. ${ }^{40} \mathrm{~A}$ special point, its null cone, and an ideal two-sphere are added at infinity to accomplish the compactification. As a result, the translations are no longer characterized by an absence of fixed points. Instead, the class of translations and the class of inverse translations each has a single fixed point (the origin and the point at infinity, respectively) while the Lorentz subgroup and the dilational subgroup leave both of these points fixed. The dilations also leave the ideal two-sphere fixed.

With these observations, we take the isotropy subgroup $\mathscr{C}_{0}$ to be the seven-dimensional homogeneous Weyl group, consisting of the six Lorentz transformations together with dilations. Defining a projection as the quotient $\mathscr{C} \mid \mathscr{C}_{0}$ we are led to a fiber bundle with an eight-dimensional manifold as the base space and the homogeneous Weyl group as a typical fiber. This will break both the translational and inverse translational symmetries when the base manifold becomes curved, but we nonetheless retain the full 15 guage field degrees of freedom.

We choose the $\mathrm{O}(4,2)$ representation of the conformal group for our notation, ${ }^{40}$ with $(A, B, \ldots)=(0,1, \ldots, 5)$. Letting boldface or Greek symbols denote forms and $(a, b, \ldots)=(1, \ldots, 4)$, the $\mathrm{O}(4,2)$ metric $\eta_{A B}$ is given by $\eta_{a b}=\operatorname{diag}(1,1,1,-1)$ and $\eta_{05}=\eta_{50}=1$ with all other compo- 
nents vanishing. Introducing the connection one-form $\omega_{B}^{A}$, we may express the covariant constancy of $\eta_{A B}$ as

$$
\mathbf{D} \eta_{A B} \equiv \mathbf{d} \eta_{A B}-\eta_{C B} \omega_{A}^{C}-\eta_{A C} \omega_{B}^{C}=0 .
$$

We may break the connection form into four independent Weyl-invariant parts: the spin connection, $\omega_{b}^{a}$, the solder form, $\omega_{0}^{a}$, the co-solder form, $\omega_{a}^{0}$, and the Weyl vector, $\omega_{0}^{0}$, where the spin connection satisfies

$$
\omega_{b}^{a}=-\eta_{b c} \eta^{a d} \omega_{d}^{c}
$$

and the remaining components of $\omega_{B}^{A}$ are given in terms of these by

$$
\begin{gathered}
\omega_{0}^{5}=\omega_{5}^{0}=0, \\
\omega_{5}^{5}=-\omega_{0}^{0}, \\
\omega_{5}^{a}=-\eta^{a b} \omega_{b}^{0}, \\
\omega_{a}^{5}=-\eta_{a b} \omega_{0}^{b} .
\end{gathered}
$$

These constraints reduce the number of independent fields $\omega_{B}^{A}$ to the required 15 and allow us to restrict $(A, B, \ldots)=(0,1, \ldots, 4)$ in all subsequent equations. Now it becomes clear why we write the metric explicitly instead of raising and lowering indices. The convention avoids confusion between the solder and co-solder forms. Staying with the $\mathrm{O}(4,2)$ index positions also indicates immediately the scaling properties of any field, since each upper index adds +1 to the conformal weight while each lower index adds -1 .

The structure constants of the conformal Lie algebra now lead immediately to the MaurerCartan structure equations of the conformal group. These are simply

$$
\mathbf{d} \omega_{B}^{A}=\omega_{B}^{C} \wedge \omega_{C}^{A} .
$$

When broken into parts based on homogeneous Weyl transformation properties, Eq. (2.10) gives

$$
\begin{gathered}
\mathbf{d} \omega_{b}^{a}=\omega_{b}^{c} \wedge \omega_{c}^{a}+\omega_{b}^{0} \wedge \omega_{0}^{a}-\eta_{b c} \eta^{a d} \omega_{d}^{0} \wedge \omega_{0}^{c}, \\
\mathbf{d} \omega_{0}^{a}=\omega_{0}^{0} \wedge \omega_{0}^{a}+\omega_{0}^{b} \wedge \omega_{b}^{a}, \\
\mathbf{d} \omega_{a}^{0}=\omega_{a}^{0} \wedge \omega_{0}^{0}+\omega_{a}^{b} \wedge \omega_{b}^{0}, \quad \mathbf{d} \omega_{0}^{0}=\omega_{0}^{a} \wedge \omega_{a}^{0} .
\end{gathered}
$$

Since no finite translation can reach the point at infinity and no inverse translation can reach the origin, the space $\mathscr{C} / \mathscr{C}_{0}$ gives a copy of (noncompact) Minkowski space for each of the two sets of translations. The generalization to a curved base space is immediate. We have

$$
\begin{aligned}
& \mathbf{d} \omega_{b}^{a}=\omega_{b}^{c} \wedge \omega_{c}^{a}+\omega_{b}^{0} \wedge \omega_{0}^{a}-\eta_{b c} \eta^{a d} \omega_{d}^{0} \wedge \omega_{0}^{c}+\Omega_{b}^{a}, \\
& \mathbf{d} \omega_{0}^{a}=\omega_{0}^{0} \wedge \omega_{0}^{a}+\omega_{0}^{b} \wedge \omega_{b}^{a}+\Omega_{0}^{a}, \\
& \mathbf{d} \omega_{a}^{0}=\omega_{a}^{0} \wedge \omega_{0}^{0}+\omega_{a}^{b} \wedge \omega_{b}^{0}+\Omega_{a}^{0}, \\
& \mathbf{d} \omega_{0}^{0}=\omega_{0}^{a} \wedge \omega_{a}^{0}+\Omega_{0}^{0} .
\end{aligned}
$$


We will call the four types of curvature $\Omega_{b}^{a}, \Omega_{0}^{a}, \Omega_{a}^{0}$, and $\Omega_{0}^{0}$ the Riemann curvature, torsion, co-torsion, and dilational curvature, respectively. Notice that if we set $\omega_{a}^{0}$, $\omega_{0}^{0}$, and the corresponding curvatures to zero, we recover Eqs. (2.2) for a four-dimensional space-time with Riemannian curvature $\Omega_{b}^{a}$ and torsion $\Omega_{0}^{a}$. If we set only $\omega_{a}^{0}=\Omega_{a}^{0}=0$, the structure equations are those of four-dimensional Weyl geometry.

We are now in a position to define a biconformal space. We state the definition for an arbitrary number of dimensions, $2 d$, although our results in all subsequent sections are given using $d=4$ for concreteness.

Definition: A biconformal space is a principal fiber bundle $(P, M, G)$ with conformal connection form $\omega=\left\{\omega_{b}^{a}, \omega_{0}^{a}, \omega_{a}^{0}, \omega_{0}^{0}\right\}(a, b=1, \ldots, d)$, where $M$ is the $2 d$-dimensional base manifold, $G$ is the Weyl group of $d$-dimensional Minkowski space, and $\pi: \omega \rightarrow\left(\omega_{0}^{a}, \omega_{a}^{0}\right)$, where $\pi$ is the canonical projection induced by $\mathscr{P} / \mathscr{G}$.

Thus, just as we can think of a space-time as a manifold and metric pair $(\mathscr{l}, g)$, we can think of a biconformal space as a manifold and connection pair $(\mathscr{l}, \omega)$. Note that in general relativity, the implicit demand for metric compatibility allows us to generate a unique connection, while at present there is no such theorem for biconformal spaces. We can go the other way, however, since the existence of a biconformal metric follows by combining the horizontal connection forms, $\left(\omega_{0}^{a}, \omega_{a}^{0}\right)$, with the Killing metric of the conformal group.

For many purposes, the starting point of computation in a biconformal space is given by the structure equations, Eqs. (2.12). Once we specify the connection, these structure equations allow us to compute the curvatures. Conversely, given horizontal curvatures satisfying the Bianchi identities [which follow from Eqs. (2.12) by differentiation], we can integrate to find the connection. Certain combinations are also possible. For example, it is easy to see from Eqs. (2.12) that in torsion-free biconformal spaces, the entire connection and the remaining curvatures are determined by $\omega_{0}^{a}$, $\omega_{a}^{0}$, and $\omega_{0}^{0}$ alone.

Horizontality requires each of the curvatures to take the form

$$
\Omega_{B}^{A}=\frac{1}{2} \Omega_{B c d}^{A} \omega_{0}^{c} \wedge \omega_{0}^{d}+\Omega_{B d}^{A c} \omega_{0}^{d} \wedge \omega_{c}^{0}+\frac{1}{2} \Omega_{B}^{A c d} \omega_{c}^{0} \wedge \omega_{d}^{0}
$$

Based on the interpretation of biconformal space as a generalization of phase space (Secs. IV and $\mathrm{V}$ below, see also Ref. 41 ) we will call $\Omega_{B c d}^{A}$ the space-time term, $\Omega_{B d}^{A c}$ the cross term, and $\Omega_{B}^{A c d}$ the momentum term of each type of curvature. In sharp contrast to the four-dimensional gauging, in which the fiber symmetry mixes the curvatures $\Omega_{b}^{a}, \Omega_{0}^{a}, \Omega_{a}^{0}$, and $\Omega_{0}^{0}$ (see Ref. 42), our choice of the homogeneous Weyl group as the structure group not only leaves these independent, but also does not mix the space-time, cross, or momentum terms. The usefulness of this type of isolation of curvature parts is evident in general relativity, where it is consistent with the bundle structure to set the torsion to zero.

Since the base space $M$ is a manifold, we can always introduce coordinates $\left(x^{\mu}, y_{\nu}\right)$ so that the connection forms may be written as

$$
\omega_{B}^{A}=\omega_{B \mu}^{A}(x, y) \mathbf{d} x^{\mu}+\omega_{B}^{A \mu}(x, y) \mathbf{d} y_{\mu} .
$$

Here the capital Latin indices range over $(0, \ldots, 4)=(0, a)$ while both Greek and lower case Latin indices range from $(1, \ldots, 4)$. The Greek indices apply to general coordinates, while the lower case Latin indices are used for the Weyl invariant parts of the orthonormal $\mathrm{O}(4,2)$ frame introduced above. Index positions indicate conformal weight, so all metrics are written explicitly. An exception to these conventions is made in Secs. III and VI where, for the flat solution, the components of the vierbein are $\delta_{\mu}^{a}$. In these sections we simply write, for example, $\left(x^{a}, y_{b}\right)$ in place of $\left(\delta_{\mu}^{a} x^{\mu}, \delta_{b}^{\nu} y_{\nu}\right)$. 


\section{FLAT BICONFORMAL SPACE}

Before deriving the form of the connection of a flat biconformal space, we discuss a few of its properties.

Definition: The connection of a flat biconformal space is said to be in the standard flat form when it is written in the following way:

$$
\begin{aligned}
& \omega_{0}^{0}=\alpha_{a}(x) \mathbf{d} x^{a}-y_{a} \mathbf{d} x^{a} \equiv W_{a} \mathbf{d} x^{a}, \\
& \omega_{0}^{a}=\mathbf{d} x^{a}, \\
& \omega_{a}^{0}=\mathbf{d} y_{a}-\left(\alpha_{a, b}+W_{a} W_{b}-\frac{1}{2} W^{2} \eta_{a b}\right) \mathbf{d} x^{b}, \\
& \omega_{b}^{a}=\left(\eta^{a c} \eta_{b d}-\delta_{d}^{a} \delta_{b}^{c}\right) W_{c} \mathbf{d} x^{d} .
\end{aligned}
$$

Notice that the Weyl vector, $W_{a}=\alpha_{a}(x)-y_{a}$, depends on an arbitrary four-vector $\alpha_{a}$ and also on the additional four coordinates $y_{a}$. The presence of $\alpha_{a}$ gives the generality required for the electromagnetic vector potential, while the $y_{a}$ keeps the dilational curvature zero. As a result, unlike Weyl's theory, the flat biconformal model predicts no size change. Also notice that the standard flat form is preserved by four-dimensional gauge transformations, $\phi(x)$, and that the gauge transformation must be associated with the undetermined vector field, $\alpha_{a}(x)$. Thus, the desirable properties of Weyl's original theory survive in this more general gauge theory.

The prediction of the exact form of the Weyl vector necessary for consistently modeling electromagnetism is nontrivial. In general, the dilational gauge vector of biconformal space is of the form

$$
\omega_{0}^{0}=\omega_{0 \mu}^{0}(x, y) \mathbf{d} x^{\mu}+\omega_{0}^{0 \mu}(x, y) \mathbf{d} y_{\mu},
$$

i.e., an eight-dimensional vector field depending on eight independent variables. Constraining the biconformal geometry to have vanishing curvatures forces $\omega_{0}^{0}=\left(\alpha_{\mu}(x)-y_{\mu}\right) \mathbf{d} x^{\mu}$. This is precisely the form required to give the Lorentz force law using the independently established formulation of Hamiltonian dynamics in biconformal space. Different field strengths $\alpha_{[\mu, \nu]}$ are in 1-1 correspondence with the possible flat biconformal spaces, so that with the interpretation of $\alpha_{\mu}$ as the vector potential, electromagnetic phenomena never lead to dilations.

We now turn to our first result.

Theorem: When the curvature of biconformal space vanishes, $\Omega_{B}^{A}=0$, there exist coordinates $\left(x^{a}, y_{a}\right)$ such that the connection takes the standard flat form. When the topology is that of $R^{8}$ the coordinates are global.

Proof: Imposing vanishing curvature, $\Omega_{B}^{A}=0$, the equations to be solved take the form

$$
\begin{aligned}
& \mathbf{d} \omega_{b}^{a}=\omega_{b}^{c} \wedge \omega_{c}^{a}+\omega_{b}^{0} \wedge \omega_{0}^{a}-\eta_{b c} \eta^{a d} \omega_{d}^{0} \wedge \omega_{0}^{c}, \\
& \mathbf{d} \omega_{0}^{a}=\omega_{0}^{0} \wedge \omega_{0}^{a}+\omega_{0}^{b} \wedge \omega_{b}^{a}, \\
& \mathbf{d} \omega_{a}^{0}=\omega_{a}^{0} \wedge \omega_{0}^{0}+\omega_{a}^{b} \wedge \omega_{b}^{0}, \\
& \mathbf{d} \omega_{0}^{0}=\omega_{0}^{a} \wedge \omega_{a}^{0} .
\end{aligned}
$$

The system may be solved by making use of the involution of Eq. (3.2b). ${ }^{38}$ This allows us to consistently set $\omega_{0}^{a}=0$ and first solve on the subspace spanned by the remaining 11 one-forms. The initial conditions for these integral submanifolds provide a coordinate $x^{a}$ such that $\omega^{a}=\mathbf{d} x^{a}$ with $x^{a}=$ const on each leaf. Each leaf is then a fiber bundle described by the simpler set of structure equations 


$$
\begin{aligned}
& \mathbf{d} \omega_{b}^{a}=\omega_{b}^{c} \wedge \omega_{c}^{a}, \\
& \mathbf{d} \omega_{a}^{0}=\omega_{a}^{0} \wedge \omega_{0}^{0}+\omega_{a}^{b} \wedge \omega_{b}^{0}, \\
& \mathbf{d} \omega_{0}^{0}=0,
\end{aligned}
$$

which may be recognized as those of a flat Weyl geometry. Equation (3.3c) implies a pure-gauge form for the Weyl vector. Choosing the gauge so that the Weyl vector vanishes (on the $\omega_{0}^{a}=0$ subspace), we are left with the structure equations for a flat Riemannian geometry. Clearly, we have the solution

$$
\omega_{b}^{a}=0, \quad \omega_{a}^{0}=\mathbf{d} y_{a}, \quad \omega_{0}^{0}=0 .
$$

Next, we reintroduce the remaining four independent one-forms,

$$
\omega_{0}^{a}=\mathbf{d} x^{a},
$$

so that $\left(x^{a}, y_{a}\right)$ provide a set of eight independent coordinates. From the linearity of the connection one-forms in the coordinate differentials, $\omega_{b}^{a}, \omega_{a}^{0}$, and $\omega_{0}^{0}$ will change only by terms proportional to $\mathbf{d} x^{a}$. We therefore may write

$$
\begin{aligned}
& \omega_{b}^{a}=C_{b c}^{a} \mathbf{d} x^{c}, \\
& \omega_{0}^{a}=\mathbf{d} x^{a}, \\
& \omega_{a}^{0}=\mathbf{d} y_{a}+B_{a b} \mathbf{d} x^{b}, \\
& \omega_{0}^{0}=W_{a} \mathbf{d} x^{a},
\end{aligned}
$$

where the coefficients $W_{a}, B_{a b}$, and $C^{a}{ }_{b c}$ are functions of $x^{a}$ and $y_{a}$ to be found by substitution into the full structure equations, Eqs. (3.2). First we determine the form of the Weyl vector, $W_{a}$, from Eq. (3.2d). Substitution of Eqs. (3.6b)-(3.6d) yields

$$
\mathbf{d} \omega_{0}^{0}=W_{d}{ }^{b} \mathbf{d} y_{b} \wedge \mathbf{d} x^{a}+W_{a, b} \mathbf{d} x^{b} \wedge \mathbf{d} x^{a}=\mathbf{d} x^{a} \wedge\left(\mathbf{d} y_{a}+B_{a b} \mathbf{d} x^{b}\right) .
$$

Here a lowered comma denotes a partial derivative with respect to $x^{b}$ while a raised comma denotes a partial with respect to $y_{b}$. Equating like components gives

$$
\begin{gathered}
W_{a}^{, b}=-\delta_{a}^{b}, \\
B_{[a b]}=W_{[b, a]} .
\end{gathered}
$$

The first of these is immediately integrated to give

$$
W_{a}=-y_{a}+\alpha_{a}(x) .
$$

Next we substitute Eqs. (3.6a) (3.6b), and (3.6d) into Eq. (3.2b) to find $C^{a}{ }_{b c}$ in terms of $W_{a}$ :

$$
\mathbf{d} \omega_{0}^{a}=0=W_{b} \mathbf{d} x^{b} \wedge \mathbf{d} x^{a}+\mathbf{d} x^{b} \wedge C^{a}{ }_{b c} \mathbf{d} x^{c}
$$

or

$$
C_{[b c]}^{a}+W_{[b} \delta_{c]}^{a}=0 .
$$


Lowering the upper index in Eq. (3.11) and noting that $C_{a b c} \equiv \eta_{a d} C^{d}{ }_{b c}=-C_{b a c}$, we add two even permutations and subtract the third to find

$$
C_{b c}^{a}=-\left(\delta_{c}^{a} \delta_{b}^{d}-\eta^{a d} \eta_{b c}\right) W_{d} .
$$

Finally, $B_{a b}$ is found from Eq. (3.2a). The substitution leads to two independent equations, one from $\mathbf{d} x^{a} \wedge \mathbf{d} y_{b}$ cross-terms and the other from terms quadratic in $\mathbf{d} x^{a}$. The first is

$$
\left(\delta_{c}^{a} \delta_{b}^{d}-\eta^{a d} \eta_{b c}\right) W_{d}{ }^{e}=\left(\eta^{a e} \eta_{b c}-\delta_{c}^{a} \delta_{b}^{e}\right),
$$

which is identically satisfied by the form of Eq. (3.9) for $W_{a}$. Writing $W^{a} \equiv \eta^{a b} W_{b}$ and $W^{2}$ $\equiv W^{a} W_{a}$, the second equation becomes

$$
\begin{aligned}
\delta_{d}^{a} B_{b c}-\delta_{c}^{a} B_{b d}-B_{d}^{a} \eta_{b c}+B_{c}^{a} \eta_{b d}= & \left(W^{a}{ }_{, c}+W^{a} W_{c}\right) \eta_{b d}-\left(W_{, d}^{a}+W^{a} W_{d}\right) \eta_{b c}-\left(W_{b, c}+W_{b} W_{c}\right) \delta_{d}^{a} \\
& +\left(W_{b, d}+W_{b} W_{d}\right) \delta_{c}^{a}-W^{2}\left(\delta_{c}^{a} \eta_{b d}-\delta_{d}^{a} \eta_{b c}\right) .
\end{aligned}
$$

Contraction on the $a$ and $c$ indices gives an expression containing $B_{a b}$ and its trace $B$ $=\eta^{a b} B_{a b}$. A second trace yields $B=W^{2}-W_{, a}^{a} . B_{a b}$ is then found to be

$$
B_{a b}=-\left(W_{a, b}+W_{a} W_{b}-\frac{1}{2} W^{2} \eta_{a b}\right) .
$$

The antisymmetric part of this expression agrees with Eq. (3.8b). The form for $B_{a b}$ above is now found to solve both Eq. (3.13) and the remaining structure equation, Eq. (3.2c), identically.

We conclude that in flat biconformal space there locally exist coordinates $x^{a}$ and $y_{a}$ such that

$$
\begin{aligned}
& \omega_{0}^{0}=\alpha_{a}(x) \mathbf{d} x^{a}-y_{a} \mathbf{d} x^{a}, \\
& \omega_{0}^{a}=\mathbf{d} x^{a}, \\
& \omega_{a}^{0}=\mathbf{d} y_{a}-\left(\alpha_{a, b}+W_{a} W_{b}-\frac{1}{2} W^{2} \eta_{a b}\right) \mathbf{d} x^{b}, \\
& \omega_{b}^{a}=-\left(\delta_{d}^{a} \delta_{b}^{c}-\eta^{a c} \eta_{b d}\right) W_{c} \mathbf{d} x^{d} .
\end{aligned}
$$

That the coordinates $\left(x^{a}, y_{a}\right)$ are global follows immediately from the $R^{8}$ topology of the base manifold, completing the proof.

We are now in a position to see in more detail how the biconformal structure corrects Weyl's theory. If we hold the $y$-coordinate constant in Eqs. (3.1), we find that Eqs. (3.1a), (3.1b), and (3.1d) are the connection forms for a four-dimensional Weyl space-time with conformally flat metric $\eta_{a b}$. The remaining expression, Eq. (3.1c), is then simply a one-form constructed from the Weyl-Ricci tensor. However, the dilational curvature of a four-dimensional Weyl geometry is given by the curl of the Weyl vector, equivalent here to the arbitrary curl of $\alpha_{a}$. Thus, when viewed from Weyl's four-dimensional perspective the solution gives unphysical size change. It is only with the inclusion of the additional momentum variables proportional to $y_{a}$ that the dilational curvature can be seen to vanish.

With the interpretation of the additional four dimensions of biconformal space as momentum variables it is clear that the actual motion of a particle is eight-dimensional. Now consider an experiment designed to detect a change in the relative size of two physical objects, for example, a pair of identical atoms. In order to see a change, a comparison must be made before and after moving the two atoms around some closed space-time loop. However, such motion necessarily involves changes in momentum as well so there is necessarily a change in $y_{a}$ as well as $x^{a}$, inducing a corresponding loop in flat biconformal space. However, no loop in flat biconformal space ever encloses nonzero dilational flux, or ever results in a measurable size change. The size 
change computed in the four-dimensional Weyl geometry is seen to be in error because it involves forcing a closed path while holding the momentum variables constant. Equivalently, the error occurs because the expression for the dilational curvature is incomplete.

We show in the next two sections that there is a direct correspondence between the geometric variables of biconformal geometry and the physical phase space variables of Hamiltonian dynamics. Then, in Sec. VI we apply the Hamiltonian correspondence to the general flat biconformal solution. This leads without further assumption to the Lorentz law of force and the identification of $\alpha_{a}$ with the electromagnetic vector potential.

\section{HAMILTONIAN DYNAMICS IN FLAT BICONFORMAL SPACE}

Leaving biconformal geometry for the moment, we turn to a geometric approach to classical Hamiltonian dynamics. We first show that the action of a classical one-particle system may be used to generate biconformal spaces. Then, in Sec. V, we give a unique prescription for generating a flat biconformal space. Begin with eight-dimensional extended phase space with canonical coordinates $\left(x^{i}, t ; p_{i}, p_{4}\right)$ where $(i, j=1,2,3)$. We assume we are given a super-Hamiltonian, $\mathscr{H}=\mathscr{H}\left(x^{a}, p_{a}\right){ }^{43}$ Imposing the constraint $\mathscr{H}=0$ then gives $p_{4}$ as a function of the remaining seven variables, $p_{4}=-H\left(p_{i}, x^{i}, t\right)$, with $H$ the usual Hamiltonian. This constraint insures that time appears as a parameter rather than an independent dynamical variable. Now consider the Hilbert form

$$
\omega=H \mathbf{d} t-p_{i} \mathbf{d} x^{i} .
$$

The integral of $\omega$ is the action functional. The exterior derivative of $\omega$ may always be factored:

$$
\mathbf{d} \omega=\frac{\partial H}{\partial x^{i}} \mathbf{d} x^{i} \wedge \mathbf{d} t+\frac{\partial H}{\partial p_{i}} \mathbf{d} p_{i} \wedge \mathbf{d} t+\mathbf{d} x^{i} \wedge \mathbf{d} p_{i}=\left(\mathbf{d} x^{i}-\frac{\partial H}{\partial p_{i}} \mathbf{d} t\right) \wedge\left(\mathbf{d} p_{i}+\frac{\partial H}{\partial x^{i}} \mathbf{d} t\right) .
$$

Therefore, if we define

$$
\begin{gathered}
\omega^{i} \equiv\left(\mathbf{d} x^{i}-\frac{\partial H}{\partial p_{i}} \mathbf{d} t\right), \quad \omega_{i} \equiv\left(\mathbf{d} p_{i}+\frac{\partial H}{\partial x^{i}} \mathbf{d} t\right), \\
\omega^{4} \equiv \mathbf{d} t, \quad \omega_{4} \equiv \mathbf{d} \mathscr{H}=0,
\end{gathered}
$$

then we can write

$$
\mathbf{d} \omega=\omega^{a} \wedge \omega_{a}=\omega^{i} \wedge \omega_{i} .
$$

This factoring is clearly preserved by local symplectic transformations of the six-basis $\left(\omega^{i}, \omega_{i}\right)$, as well as reparametrizations of the time. Obviously these transformations include the usual canonical transformations of coordinates as a special case. One class of such allowed transformations of basis is achieved by the addition of $c_{a b} \omega^{b}$ to $\omega_{a}$, where $c_{a b}=c_{b a}$. For the moment we take $c_{a b}=0$, but below we show the existence of a unique choice of $c_{a b}$ that leads to a flat biconformal space.

We may also define a connection one-form, $\omega_{b}^{a}$. This choice is uniquely determined by requiring the resulting biconformal space to be flat, but first we show that every possible choice leads to some biconformal space. Without imposing flatness any choice is possible. For this most general case, let $\omega_{b}^{a}$ be any linear combination of $\omega_{0}^{a}$ and $\omega_{a}^{0}$, and define the (necessarily horizontal) curvatures to be

$$
\Omega_{b}^{a} \equiv \mathbf{d} \omega_{b}^{a}-\omega_{b}^{c} \wedge \omega_{c}^{a}-\omega_{b}^{0} \wedge \omega_{0}^{a}-\eta_{b c} \eta^{a d} \omega_{0}^{c} \wedge \omega_{d}^{0}
$$




$$
\begin{gathered}
\Omega_{0}^{a} \equiv \mathbf{d} \omega_{0}^{a}-\omega \wedge \omega_{0}^{a}-\omega_{0}^{b} \wedge \omega_{b}^{a}, \\
\Omega_{a}^{0} \equiv \mathbf{d} \omega_{a}^{0}-\omega_{a}^{0} \wedge \omega-\omega_{a}^{b} \wedge \omega_{b}^{0}, \\
\Omega_{0}^{0} \equiv \mathbf{d} \omega-\omega_{0}^{a} \wedge \omega_{a}^{0} \equiv 0 .
\end{gathered}
$$

The necessary presence and form of Eq. (4.4) for $\mathbf{d} \omega$, and the dependence of the curvatures on both $\omega^{a}$ and $\omega_{a}$ clearly show this to be a dilationally flat (i.e., $\Omega_{0}^{0}=0$ ) biconformal space. Therefore, extended phase space together with a Hamiltonian symplectic structure may be viewed as a certain kind of biconformal space. Turning this around, we can interpret biconformal space, and therefore conformal gauge theory, as a generalization of one-particle phase space.

It is worth pointing out that biconformal spaces, while including Hamiltonian extended phase spaces as special cases, also contain all four-dimensional pseudo-Riemannian geometries as other special cases. We therefore have a differential geometry rich enough to describe both general relativity and Hamiltonian particle dynamics. Furthermore, as we show in Sec. VII, biconformal spaces include the even larger class of all four-dimensional Weyl geometries, and this class allows the consistent geometric unification of gravity and electromagnetism.

The presence of both Hamiltonian and Riemannian structures is reassuring, since it is one aim of the study of biconformal spaces to place relativity theory and quantum theory in a common mathematical framework. While the developments here suggest only the possibility of describing, perhaps, a quantum particle in a curved background, the full picture is actually somewhat better because the symplectic two-form of the Hamiltonian structure gives a complex structure to the tangent/cotangent space and an almost complex structure to the biconformal space itself. The biconformal structure therefore gives a natural complexification of space-time in such a way that the usual real structure is immediately evident. One might hope, for example, to see some special significance for the Ashtekar connection when biconformal space is expressed in terms of the $\mathrm{SU}(2,2)$ conformal covering group instead of $\mathrm{O}(4,2)$. Whether these hopes are realized or not is the subject of current study.

We also note here that the idea of a quantum interpretation of conformal geometry agrees in some ways with earlier proposals ${ }^{44-47}$ relating phase space, Weyl geometry, and quantum physics. These proposals, however, lack the full geometric structure of conformal gauge theory, do not demonstrate the intrinsically biconformal structure of Hamiltonian systems, and use a different inner product than that proposed in Sec. VI (see also Ref. 41).

We next show the relationship of the geometry described by Eqs. (4.5) to classical mechanics.

Since the curvatures are two-forms and because $\mathbf{d} \mathscr{H}=0$, each of the curvatures, $\Omega^{a}$ and $\Omega_{a}$, is necessarily at least linear in one of the six basis forms $\left(\omega^{i}, \omega_{i}\right)$. Thus, six of the structure equations, Eqs. (4.5b) and (4.5c), are in involution. This involution allows us to set $\omega^{i}=\omega_{i}=0$, thereby singling out a fibration of the bundle by one-dimensional submanifolds, i.e., the classical paths of motion. Examination of Eq. (4.3) shows that these conditions simply give Hamilton's equations of motion:

$$
\mathbf{d} x^{i}=\frac{\partial H}{\partial p_{i}} \mathbf{d} t, \quad \mathbf{d} p_{i}=-\frac{\partial H}{\partial x^{i}} \mathbf{d} t
$$

The Fröbenius theorem guarantees the existence of solutions to these equations for the paths. The remaining two structure equations then reduce to

$$
\mathbf{d} \omega_{j}^{i}=\mathbf{d} \omega=0,
$$

which are identically satisfied on curves. 
It is of interest to further note that on the full bundle the condition that $\omega$ be exact is the Hamiltonian-Jacobi equation, since we may then write $\omega=\mathbf{d} S$. Substituting for $\omega$ and expanding d $S$ gives

$$
-H \mathbf{d} t+p_{i} \mathbf{d} x^{i}=\frac{\partial S}{\partial x^{i}} \mathbf{d} x^{i}+\frac{\partial S}{\partial p_{a}} \mathbf{d} p_{a}+\frac{\partial S}{\partial t} \mathbf{d} t,
$$

so that

$$
\begin{gathered}
\frac{\partial S}{\partial p_{i}}=0, \quad \frac{\partial S}{\partial p_{4}}=0 \\
\frac{\partial S}{\partial x^{i}}=p_{i}
\end{gathered}
$$

and

$$
H\left(\frac{\partial S}{\partial x^{i}}, x^{i}, t\right)=-\frac{\partial S}{\partial t}
$$

Therefore, since $\omega$ is the Weyl vector of the generated biconformal space, the Hamilton-Jacobi equation holds if and only if the Weyl vector is pure gauge, $\omega=\mathbf{d} S$. A gauge transformation reduces the Weyl vector to zero. Since the dilational curvature is always zero for the geometry built from a Hamiltonian system, when $\mathbf{d} \omega=0$ we also have

$$
\omega^{i} \wedge \omega_{i}=0
$$

implying three linear dependences between these six solder forms. Together with the vanishing of d $\mathscr{H}$, the Hamilton-Jacobi equation therefore specifies a four-dimensional subspace of the full biconformal space.

\section{FLAT BICONFORMAL SPACE AND THE HAMILTONIAN GEOMETRY}

In this section, we show how to specify a unique flat biconformal space for a given Hamiltonian system. This is achieved by making a judicious choice of the solder and co-solder forms.

This time we initially regard the super-Hamiltonian as an unconstrained function of all eight coordinates. The Hamiltonian, $H$, is again defined as the solution for $-p_{4}$ when $\mathscr{H}$ is zero. Thus, when $\mathscr{H}$ is unconstrained, $p_{4}$ is also variable and the Hilbert form generalizes to

$$
\omega_{0}^{0}=-p_{a} \mathbf{d} x^{a}=-p_{4}(\mathscr{H}, H) \mathbf{d} t-p_{i} \mathbf{d} x^{i} .
$$

Then

$$
\mathbf{d} \omega_{0}^{0}=\mathbf{d} x^{a} \wedge \mathbf{d} p_{a},
$$

where we reserve the symbols $\omega, \omega_{i}$, and $\omega^{i}$ for the special case when $\mathscr{H}=0$ and $p_{4}$ $=-H\left(x^{i}, t, p_{i}\right)$.

The solder and co-solder forms $\omega_{0}^{a}$ and $\omega_{a}^{0}$ are now identified by comparing the expression for $\mathbf{d} \omega_{0}^{0}$ to the flat biconformal solution, Eqs. (3.1). To make Eq. (3.1a) agree with Eq. (5.1) we must have $\alpha_{a}(x)=0$ and $y_{a}=p_{a}$. We can set $\alpha_{a}=0$ with a gauge change as long as $\alpha_{[a, b]}=0$. Then, with $y_{a}=p_{a}$, the remainder of the connection is fully determined to be

$$
\omega_{0}^{0}=-p_{a} \mathbf{d} x^{a}, \quad \omega_{0}^{a}=\mathbf{d} x^{a},
$$




$$
\begin{gathered}
\omega_{a}^{0}=\mathbf{d} p_{a}-\left(p_{a} p_{b}-\frac{1}{2} p^{2} \eta_{a b}\right) \mathbf{d} x^{b}, \\
\omega_{b}^{a}=-\left(\delta_{d}^{a} \delta_{b}^{c}-\eta^{a c} \eta_{b d}\right) p_{c} \mathbf{d} x^{d} .
\end{gathered}
$$

Note that canonical changes of variables do not change $\omega_{0}^{0}$ by more than a scale change, $\mathbf{d} \phi$, so this form of the connection is correct for any canonical variables $\left(x^{i}, p_{i}\right) \rightarrow\left(q^{i}, \pi_{i}\right)$.

We now restrict to the hypersurface $\mathscr{H}=0$ so that $p_{4}=-H\left(p_{i}, x^{i}, t\right)$. The symmetric coefficient

$$
c_{a b}=p_{a} p_{b}-\frac{1}{2} p^{2} \eta_{a b}
$$

provides a symplectic change of basis with respect to the manifestly closed and nondegenerate two-form, $\mathbf{d} \omega_{0}^{0}=\omega_{0}^{a} \wedge \omega_{a}^{0}=\mathbf{d} x^{a} \wedge \mathbf{d} p_{a}$. The differential of $\omega_{0}^{0}$ is still seen to factor as in Eq. (4.2) either directly by differentiation or by substitution of $p_{4}=-H\left(p_{i}, x^{i}, t\right)$ into $\omega_{0}^{a} \wedge \omega_{a}^{0}$, with $\omega_{0}^{a}$ and $\omega_{a}^{0}$ given by Eqs. (5.3). The involution of Eqs. (4.5b) and (4.5c) for $\omega_{i}$ and $\omega^{i}$ still holds, with the classical curves given by $\omega_{i}=\omega^{i}=0$. We therefore recover the Hamiltonian system, and have shown it to lie in a unique flat biconformal space.

The resulting curves in the biconformal space are easily found by first writing the frame field in terms of $\omega^{i}$ and $\omega_{i}$ :

$$
\begin{aligned}
& \omega_{0}^{0}=\left(H-p_{i} \frac{\partial H}{\partial p_{i}}\right) \mathbf{d} t-p_{i} \omega^{i}=L\left(\dot{x}^{i}, x^{i}, t\right) \mathbf{d} t-p_{i} \omega^{i}, \\
& \omega_{0}^{i}=\omega^{i}+\frac{\partial H}{\partial p_{i}} \mathbf{d} t, \\
& \omega_{0}^{4}=\mathbf{d} t, \\
& \omega_{i}^{0}=\omega_{i}-\left(p_{i} p_{j}+\frac{1}{2}\left(H^{2}-p^{2}\right) \delta_{i j}\right) \omega^{j}+\left(p_{i} H-\frac{\partial H}{\partial x^{i}}-\frac{1}{2}\left(H^{2}-p^{2}\right) \delta_{i j} \frac{\partial H}{\partial p_{j}}\right) \mathbf{d} t, \\
& \omega_{4}^{0}=\left(H p_{i}-\frac{\partial H}{\partial x^{i}}\right) \omega^{i}+\left(H \frac{\partial H}{\partial p_{i}} p_{i}-\frac{1}{2}\left(H^{2}-p^{2}\right)-\frac{\partial H}{\partial x^{i}} \frac{\partial H}{\partial p_{i}}\right) \mathbf{d} t, \\
& \omega_{j}^{i}=-\left(\delta_{l}^{i} \delta_{j}^{k}-\delta^{i k} \eta_{j l}\right) p_{k}\left(\omega^{l}+\frac{\partial H}{\partial p_{l}} \mathbf{d} t\right), \\
& \omega_{4}^{i}=H \omega^{i}-\left(\delta^{i j} p_{j}-H \frac{\partial H}{\partial p_{i}}\right) \mathbf{d} t, \\
& \omega_{i}^{4}=-\delta_{i j} \omega_{4}^{j} .
\end{aligned}
$$

The simple example of a free particle is instructive. Setting $\omega^{i}=\omega_{i}=0$ and $H^{2}=p^{2}+m^{2} \neq 0$, Eqs. (5.5) reduce to

$$
\begin{gathered}
\omega_{0}^{0}=\frac{m^{2}}{H} \mathbf{d} t=m \mathbf{d} \tau, \quad \omega_{0}^{a}=\frac{\eta^{a b} p_{b}}{m^{2}} \omega_{0}^{0}=u^{a} \mathbf{d} \tau=\mathbf{d} x^{a}, \\
\omega_{a}^{0}=\frac{1}{2} p_{a} \omega_{0}^{0}=\frac{1}{2} m p_{a} \mathbf{d} \tau, \quad \omega_{b}^{a}=0,
\end{gathered}
$$


where use of $\omega_{0}^{0}$ or proper time $\mathbf{d} \tau$ in place of $\mathbf{d} t$ simplifies the expressions. The solder and co-solder forms are proportional to the displacement and momentum, respectively.

Also, we see again that the involution required to specify the classical paths necessarily exists. Since the biconformal base space is spanned by the eight forms $\mathbf{d} x^{a}$ and $\mathbf{d} p_{a}$, the only way the involution could fail is if there was an independent $\mathbf{d} t \wedge \mathbf{d} p_{4}$ term in the torsion or co-torsion of the $p_{4}=-H$ hypersurface. However, since $\mathbf{d} H$ is given a priori in terms of the other seven forms, this cannot happen. We conclude that the Hamiltonian dynamics of a point particle is equivalent to the specification of a hypersurface, $y_{4}=y_{4}\left(y_{i}, x^{a}\right)$, in a flat biconformal space, and the consequent necessary existence of a preferred congruence of curves in the hypersurface.

The principal significance of this result is the unambiguous identification of the geometric quantities which arise in the gauging of the 15-dimensional conformal group with corresponding physical quantities in phase space. In particular, the extra four coordinates are identified with momenta and the integral of the Weyl vector is identified with the action. This insight should not be construed as a replacement for the phase space description of particle mechanics. Rather, the interpretation presented here is to be regarded as the one-particle limit of a full biconformal field theory, and is intended to provide guidance for and a check on that field theory. We shall see in Sec. VII that for curved biconformal spaces, the "momentumlike" basis forms $\omega_{a}^{0}$ contain the stress-energy source for the Einstein equation, further strengthening the present interpretation.

Notice that for multiparticle systems this interpretation of the variables of flat biconformal space in terms of phase space variables differs from the usual $n$-particle phase space of more complicated systems. In the case of multiple particles in a small region of (nearly) flat biconformal space, the particles share both the momentum and configuration space. Thus, while a single point of a multiparticle phase space characterizes the entire multiparticle system, the many particle biconformal model will be described by many points in the same eight-dimensional space. Nonetheless, the physical interpretation of the extra four coordinates as momenta remains valid, and each particle treated separately will (locally) obey its own set of Hamilton's equations.

The same conclusion holds for the fields in biconformal spaces-the extra dimensions will give the local field momentum. This may be seen in either of two ways. First, fields are the continuum limits of multiparticle systems and their local momentum will therefore be the limit of the particle momenta. Second, whenever a single field quantum is confined to an isolated region which is small relative to the curvature, it will have an interpretation as a single particle. The biconformal co-space must then give the momentum of that particle.

While accomplishing an interpretation of the biconformal variables, we have also circumvented problems with previous treatments of conformal gauge theory. Unlike four-dimensional conformal gaugings which always reduce to a four-dimensional Weyl geometry in which the inverse translations are auxiliary, the current approach retains the full conformal degrees of freedom. The extra four degrees of freedom are now seen to correspond to the inclusion of momentum variables in the physical description.

\section{WEYL'S THEORY IN BICONFORMAL SPACE}

Now consider how the dynamics of the Hamiltonian correspondence of Secs. IV and V is modified by the presence of the vector field, $\alpha$. The Weyl vector is now given by

$$
\omega_{0}^{0}=-p_{a} \mathbf{d} x^{a}+\alpha_{a} \mathbf{d} x^{a} \equiv-\pi_{a} \mathbf{d} x^{a}
$$

so that

$$
\mathbf{d} \omega_{0}^{0}=\mathbf{d} x^{a} \wedge \mathbf{d} \pi_{a} .
$$

Specifying $\pi_{4}=\pi_{4}\left(\pi_{i}, x^{a}\right)$ and setting $\omega^{i}=\omega_{i}=0$ again leads to Hamilton's equations, in the form 


$$
\begin{gathered}
\mathbf{d} x^{i}=-\frac{\partial \pi_{4}}{\partial \pi_{i}} \mathbf{d} t, \\
\mathbf{d} p_{i}=\frac{\partial \pi_{4}}{\partial x^{i}} \mathbf{d} t .
\end{gathered}
$$

Maintaining our previous identification $p_{4}=-H=-\left(m^{2}+p^{2}\right)^{1 / 2}$ we find

$$
\pi_{4}=-\left(m^{2}+p^{2}\right)^{1 / 2}-\alpha_{4}=-\left(m^{2}+\left(\pi^{i}+\alpha^{i}\right)\left(\pi_{i}+\alpha_{i}\right)\right)^{1 / 2}-\alpha_{4} .
$$

Hamilton's equations become

$$
\mathbf{d} x^{i}=\frac{\pi^{i}+\alpha^{i}}{\left(m^{2}+(\pi+\alpha)^{2}\right)^{1 / 2}} \mathbf{d} t=\frac{p^{i}}{\left(m^{2}+p^{2}\right)^{1 / 2}} \mathbf{d} t
$$

or

$$
\dot{x}^{i}=\frac{p^{i}}{\left(m^{2}+p^{2}\right)^{1 / 2}}
$$

for the position variables and

$$
\mathbf{d} \pi_{i}=-\frac{\partial \alpha_{4}}{\partial x^{i}} \mathbf{d} t-\frac{\pi^{j}+\alpha^{j}}{\left(m^{2}+(\pi+\alpha)^{2}\right)^{1 / 2}} \alpha_{j, i} \mathbf{d} t=-\frac{\partial \alpha_{4}}{\partial x^{i}} \mathbf{d} t-\dot{x}^{j} \alpha_{j, i} \mathbf{d} t
$$

for the momentum. The left-hand side of Eq. (6.6) expands to

$$
\mathbf{d} \pi_{i}=\mathbf{d} p_{i}-\frac{\partial \alpha_{i}}{\partial t} \mathbf{d} t-\alpha_{i, j} \mathbf{d} x^{j},
$$

which finally leads to

$$
\frac{d p_{i}}{d t}=\left(-\frac{\partial \alpha_{4}}{\partial x^{i}}+\frac{\partial \alpha_{i}}{\partial t}\right)+\dot{x}^{j}\left(\alpha_{i, j}-\alpha_{j, t}\right)
$$

For the time component we have

$$
\begin{aligned}
\frac{d p_{4}}{d t} & =\frac{d}{d t}\left(\pi_{4}+\alpha_{4}\right)=\frac{d}{d t}\left(-\left(m^{2}+p^{2}\right)^{1 / 2}\right) \\
& =-\frac{1}{\left(m^{2}+p^{2}\right)^{1 / 2}} p^{i} \frac{d p_{i}}{d t}=-\dot{x}^{i} \frac{d p_{i}}{d t}=\dot{x}^{i}\left(\frac{\partial \alpha_{4}}{\partial x^{i}}-\frac{\partial \alpha_{i}}{\partial t}\right) .
\end{aligned}
$$

Equations (6.7) give the Lorentz force law if we identify

$$
\alpha_{a}=q\left(\phi,-A_{i}\right)=-q A_{a} .
$$

Thus, the existence and form of the electromagnetic force on a charged particle is correctly predicted by the general solution for flat biconformal space. The presence of the vector field $\alpha_{a}$, its gauge dependence, and its proper coupling to matter are direct consequences of the local gauge theory of scalings and of our identification of Hamiltonian dynamics as the specification of a 
hypersurface in flat biconformal space. Nor does this formulation suffer the objection made to the original Weyl theory. Since we are studying precisely the flat biconformal spaces, there is no dilational curvature and no measurable size change.

In concluding this section, we comment briefly on the relationship of these considerations to the standard U(1) model of electromagnetic field theory. Biconformal space has a natural metric structure based on the Killing metric of the underlying conformal group. The Killing metric has eigenvalues \pm 1 and zero signature, hence the local form

$$
K_{\mathscr{C}, \mathscr{B}}=\operatorname{diag}\left(\eta_{a b},-\eta^{a b}\right),
$$

where $(\mathscr{C}, \mathscr{B})=(1, \ldots, 8)$. Consequently, the proportionality constant in the identification between $y_{a}$ and $p_{a}$ is purely imaginary. ${ }^{48}$ Since the vector field $\alpha_{a}(x)$ is real, we have

$$
\begin{aligned}
y_{a} & =\lambda\left(i p_{a}+\alpha_{a}\right) \\
& =i \lambda\left(p_{a}-i \alpha_{a}\right)
\end{aligned}
$$

as the relationship between geometric and physical variables. Since the proportionality constant drops out of Hamilton's equations it is not measurable classically, but it must be included whenever particle paths are allowed to deviate from the classical trajectories. Thus, the real-valued four-dimensional scale invariance that preserves the form of the standard flat biconformal connection becomes a U(1) invariance when applied to the physical variables of Eq. (6.9b).

\section{COUPLING TO GRAVITY AND OTHER FIELDS}

So far, we have motivated a new eight-dimensional gauging of the conformal group, found the class of flat solutions of the resulting biconformal spaces, and shown how to interpret the flat solutions as a phase space for a single particle coupled to a background electromagnetic field. In this section, we generalize these results to gravitating biconformal spaces in which the solder form satisfies the Einstein equation with arbitrary matter as source, and the vector potential identified in the previous sections satisfies the Maxwell field equations with arbitrary electromagnetic currents. The results of this section therefore comprise a unified geometric theory of gravitation and electromagnetism.

Our starting point is the full set of structure equations, Eqs. (2.12), which define the 15 curvature two-forms $\Omega_{B}^{A}$. Each of these curvatures has the three biconformally invariant terms displayed in Eq. (2.13). In addition, we note that the two-form $\mathbf{d} \omega_{0}^{0}$ is separately biconformally invariant, and the solder and co-solder forms $\omega_{0}^{a}$ and $\omega_{a}^{0}$ transform tensorially. As long as the corresponding Bianchi identities are satisfied, a specification of any combination of these fields may be used to invariantly determine subclasses of biconformal geometries.

To begin, we seek some general constraints to limit the number of independent fields. For classical geometries it is reasonable to assume that no classical path in phase space encloses a plaquette on which the dilation, $\Omega_{0}^{0}$, is nonvanishing. The simplest (but by no means the only) way to guarantee this is to just set $\Omega_{0}^{0}=0$. We also expect that the space-time torsion will vanish in typical classical models, and again make the simplest hypothesis, that the full torsion (but not the co-torsion) is zero. Thus we have the general constraints

$$
\begin{aligned}
& \Omega_{0}^{0}=0, \\
& \Omega_{0}^{a}=0 .
\end{aligned}
$$


Next, we note that the vanishing of the torsion puts the solder form in involution. Assuming the resulting foliation to be regular, there exists a four-dimensional submanifold of the base space spanned by $\omega_{0}^{a}=\mathbf{e}^{a}$. As a final general constraint, we require the existence of a completion $\mathbf{f}_{a}$ to the $\mathbf{e}^{a}$ basis in which the space-time curvature is traceless:

$$
\Omega_{b a c}^{a}=0 \quad\left[\text { in the }\left(\mathbf{e}^{a}, \mathbf{f}_{a}\right) \text { basis }\right] .
$$

We shall show that these three constraints are sufficient for the resulting class of geometries to take a recognizable form. There is no restriction of the dependence of any of the fields on the eight coordinates, $\left(x^{\mu}, y_{\nu}\right)$. Notice that we now return to the use of Greek indices for general coordinates.

In addition to the general constraints above, we posit two field equations. The first provides a source for the Weyl vector, which we take here to be in the typical form for an electromagnetic current

$$
* \mathbf{d} * \mathbf{d} \omega_{0}^{0}=\mathbf{J}=J_{a}(x) \mathbf{e}^{a} .
$$

Finally, part of the co-solder form is determined by an arbitrary stress-energy tensor $T_{a b}$ via

$$
\omega_{a}^{0}=\mathscr{T}_{a}+\cdots,
$$

where $\mathscr{T}_{a} \equiv-\frac{1}{2}\left(T_{a b}-\frac{1}{3} \eta_{a b} T\right) \mathbf{e}^{b}$. The consistency of these expressions and the form of the remaining part of the co-solder form are established using the general equations, Eqs. (7.1)-(7.3), together with the structure equations, Eqs. (2.12).

The central result of this section is that a biconformal space is in 1-1 correspondence with a four-dimensional Einstein-Maxwell space-time if and only if Eqs. (7.1)-(7.4) hold. The 1-1 correspondence is based on an isomorphism between the biconformal co-space at $x_{0}^{\mu}$ and the tangent space to a four-dimensional space-time at a corresponding point $x_{0}^{\mu}$. To see roughly how this isomorphism occurs, note that the biconformal base space is spanned by the differentials $\left(\mathbf{d} x^{\mu}, \mathbf{d} y_{\nu}\right)$. Setting $x^{\mu}=x_{0}^{\mu}$ gives $\mathbf{d} x_{0}^{\mu}=0$, thereby restricting to the subspace spanned by $\mathbf{d} y_{\nu}$. We will show that the conditions above make the biconformal space flat in the $y$ variables, giving a four-dimensional Minkowski space with basis $\mathbf{d} y_{\nu}$. Then, because the basis $\mathbf{d} y_{\nu}$ transforms oppositely under Lorentz transformations from $\mathbf{d} x^{\mu}$, and because the $\mathbf{d} x^{\mu}$ spans the cotangent space of an orthogonal four-dimensional submanifold, there exists an isomorphism between the $\mathbf{d} y_{\nu}$ space and the tangent space of the submanifold. A more complete presentation of the techniques used and some related results are given in Ref. 49.

The equations to be solved are

$$
\begin{aligned}
& \mathbf{d} \omega_{b}^{a}=\omega_{b}^{c} \wedge \omega_{c}^{a}+\omega_{b}^{a} \wedge \omega_{0}^{a}-\eta_{b c} \eta^{a d} \omega_{d}^{0} \wedge \omega_{0}^{c}+\Omega_{b}^{a}, \\
& \mathbf{d} \omega_{0}^{a}=\omega_{0}^{0} \wedge \omega_{0}^{a}+\omega_{0}^{b} \wedge \omega_{b}^{a}, \\
& \mathbf{d} \omega_{a}^{0}=\omega_{a}^{0} \wedge \omega_{0}^{0}+\omega_{a}^{b} \wedge \omega_{b}^{0}+\Omega_{a}^{0}, \\
& \mathbf{d} \omega_{0}^{0}=\omega_{0}^{a} \wedge \omega_{a}^{0} .
\end{aligned}
$$

The first part of our proof follows Theorem I of Ref. 49. We begin with the Bianchi identity for Eq. $\left(2.12 b^{\prime}\right)$, which follows by taking the exterior derivative and using $\mathbf{d}^{2}=0$. The result is

$$
\omega_{0}^{b} \wedge \Omega_{b}^{a}=0,
$$

which in particular shows that the momentum term of the curvature vanishes 


$$
\Omega_{b}^{a c d}=0
$$

Similarly, the Bianchi identity for Eq. (2.12d') requires

$$
\Omega_{b}^{0 c d}=0
$$

for the co-torsion. Together with the vanishing of the dilation and the torsion, Eqs. (7.6) and (7.7) imply that the bundle is momentum flat, i.e., the momentum term of each curvature vanishes.

Next, we use the involution of the solder form, as noted above. The involution means that the biconformal bundle is foliated by 11-dimensional subbundles on which $\omega_{0}^{a}=0$. On this subbundle, using the momentum-flatness, the structure equations reduce to

$$
\begin{aligned}
& \mathbf{d} \omega_{b}^{a}=\omega_{b}^{c} \wedge \omega_{c}^{a}, \\
& \mathbf{d} \omega_{a}^{0}=\omega_{a}^{0} \wedge \omega_{0}^{0}+\omega_{a}^{b} \wedge \omega_{b}^{0}, \\
& \mathbf{d} \omega_{0}^{0}=0 .
\end{aligned}
$$

Equations (7.8) are just the structure equations for a flat four-dimensional Weyl geometry. Equation (7.8a) shows that we can perform a Lorentz gauge transformation on the entire bundle such that $\left.\omega_{b}^{a}\right|_{\omega_{0}^{a}=0}=0$, while Eq. (7.8c) shows the existence of a scaling such that $\left.\omega_{0}^{0}\right|_{\omega_{0}^{a}=0}=0$. Following these gauge changes Eq. (7.8b) takes the simple form

$$
\mathbf{d} \omega_{a}^{0}=0
$$

with the immediate solution

$$
\left.\omega_{a}^{0}\right|_{\omega_{0}^{a}=0}=f_{a}^{\mu}(x) \mathbf{d} y_{\mu}
$$

where we have introduced four coordinates $y_{\mu}$ to span the four-dimensional base space of the submanifold. Notice that the coefficient matrix $f_{a}^{\mu}(x)$ must be nondegenerate.

Now, assuming the foliation to be regular, the space of leaves of the foliation is also a four-dimensional manifold spanned by $\omega_{0}^{a}$. There exist coordinates $x^{\mu}$ such that

$$
\omega_{0}^{a}=e_{\mu}^{a}(x, y) \mathbf{d} x^{\mu}=\mathbf{e}^{a}
$$

so that each leaf of the foliation is given by some constant value $x^{\mu}=x_{0}^{\mu}$.

From the subbundle, with connection $\left.\omega_{b}^{a}\right|_{x_{0}}=0,\left.\omega_{0}^{0}\right|_{x_{0}}=0$, and $\left.\omega_{a}^{0}\right|_{x_{0}}=\mathbf{f}_{a}$, we extend back to the full bundle by allowing $x$ to vary. This can change each connection form at most by a term proportional to $\mathbf{d} x^{\mu}$ or equivalently $\mathbf{e}^{a}$, so the full connection may always be given the local form

$$
\begin{gathered}
\omega_{b}^{a}=C_{b c}^{a} \mathbf{e}^{c}, \\
\omega_{0}^{a}=\mathbf{e}^{a}(x, y), \\
\omega_{a}^{0}=f_{a}^{\mu}(x) \mathbf{d} y_{\mu}+B_{a b} e^{b} \equiv \mathbf{f}_{a}+\mathbf{B}_{a}, \\
\omega_{0}^{0}=W_{a} \mathbf{e}^{a},
\end{gathered}
$$

where $W_{a}, B_{a b}$, and $C_{b c}^{a}$ are functions of all eight coordinates $\left(x^{\mu}, y_{\nu}\right)$. The functional dependence of $e_{\mu}{ }^{a}$, while a priori unspecified, is actually restricted by Eq. $\left(2.12 \mathrm{~b}^{\prime}\right)$. Since $\omega_{b}^{a}$ and $\omega_{0}^{0}$ 
depend only on $\mathbf{e}^{a}$ and not $\mathbf{f}_{a}$, writing out $\mathbf{d e}^{a}$ in coordinates immediately shows that $\partial^{\nu} e_{\mu}{ }^{a}=0$, where we write $\partial^{\nu} \equiv \partial / \partial y_{\nu}$ and $\partial_{\nu} \equiv \partial / \partial x^{\nu}$. Therefore, we may drop the $y$ dependence in Eq. (7.11b).

If we had made a different initial choice of the coordinates $y_{\mu}$, the connection would still be of the form given by Eqs. (7.11), although $W_{a}, B_{a b}$, and $C_{b c}^{a}$ would be different functions. We can therefore assume without loss of generality that the basis $\left(\mathbf{e}^{a}, \mathbf{f}_{b}\right)$ has been chosen as the one in which the space-time curvature is tracefree.

Next, after dividing $B_{a b}$ into symmetric and antisymmetric parts,

$$
B_{a b} \equiv S_{a b}(x)+F_{a b}(x)
$$

with $S_{a b}=S_{(a b)}$ and $F_{a b}=F_{[a b]}$, we substitute Eq. (7.11c) into the dilation equation, Eq. (2.12d'), to find

$$
\mathbf{d} \omega_{0}^{0}=\mathbf{e}^{a} \wedge \mathbf{f}_{a}+\mathbf{F}=\mathbf{d} x^{\mu} \wedge f_{\mu}{ }^{\nu}(x) \mathbf{d} y_{\nu}+\mathbf{F},
$$

where $\mathbf{F} \equiv F_{a b} \mathbf{e}^{a} \wedge \mathbf{e}^{b}=F_{\mu \nu} \mathbf{d} x^{\mu} \wedge \mathbf{d} x^{\nu}$ and $f_{\mu}{ }^{\nu} \equiv e_{\mu}{ }^{a} f_{a}{ }^{\nu}$. The mixed terms of Eq. (7.13) yield

$$
W_{\mu}{ }^{, \nu}=-f_{\mu}^{\nu}(x),
$$

which is immediately integrated to give

$$
\omega_{0}^{0} \equiv W_{\mu} \mathbf{d} x^{\mu}=\left(-f_{\mu}{ }^{\nu}(x) y_{\nu}+A_{\mu}(x)\right) \mathbf{d} x^{\mu}
$$

up to a gauge transformation. Here $A_{\mu}(x)$ is an arbitrary integration constant for the $y$ integration.

We could continue to carry the field $f_{\mu}{ }^{\nu}(x)$ through the remainder of the general constraints, but it is simpler to employ the field equation to remove it now. In a more general class of models, $f_{\mu}^{\nu}(x)$ provides an additional unspecified set of fields. While it is easy to conjecture that these extra fields may lead to a geometric electroweak theory, $f_{\mu}{ }^{\nu}(x)$ appears on the surface to be a translational rather than a rotational Yang-Mills field, so that the required quadratic terms are missing from the field strength. The proper role of these fields is under study. For our present purpose, we simply note that the field equation

$$
* \mathbf{d} * \mathbf{d} \omega_{0}^{0}=\mathbf{J}=J_{a}(x) e^{a}
$$

has no source for the part of $\omega_{0}^{0}$ linear in $y$, and therefore gives

$$
\left[* \mathbf{d} * \mathbf{d} f_{\mu}{ }^{\nu}(x)\right] y_{\nu}=0
$$

for the $y$-dependent part of the space-time term. This is a four-dimensional electromagnetic-type equation for the "potential" $f_{\mu} \equiv f_{\mu}{ }^{\nu}(x) y_{\nu}$, which has the unique solution $f_{\mu}{ }^{\nu}(x)=0$ for vanishing boundary conditions at infinity. To this solution we add the particular solution $f_{\nu}{ }^{\nu}=$ const, since $f_{\mu}{ }^{\nu}(x)$ must be invertible as noted above. Finally, a constant change of the $y$ coordinates gives $f_{\mu}{ }^{\nu}=\delta_{\mu}^{\nu}$, simplifying the co-solder form to

$$
\omega_{a}^{0}=e_{a}{ }^{\mu}(x) \mathbf{d} y_{\mu}+B_{a \mu}(x, y) \mathbf{d} x^{\mu},
$$

where $e_{a}{ }^{\mu}$ is the inverse to $e_{\mu}{ }^{a}$.

With the solder form given by Eq. (7.17), Eq. (7.15) becomes

$$
\omega_{0}^{0} \equiv W_{\mu} \mathbf{d} x^{\mu}=\left(-y_{\mu}+A_{\mu}(x)\right) \mathbf{d} x^{\mu} .
$$


Thus, this entire class of geometries has the same "minimal coupling" form of the Weyl vector as we found for the flat case. Notice also that the form of Eq. (7.18) is not affected by a purely $x$-dependent gauge transformation, which is consistent with the interpretation of $\mathbf{A}$ as the electromagnetic vector potential.

Next, we move to Eq. $\left(2.12 b^{\prime}\right)$, which becomes

$$
\mathbf{d e} \mathbf{e}^{a}=\omega_{0}^{0} \wedge \mathbf{e}^{a}+\mathbf{e}^{b} \wedge \omega_{b}^{a} .
$$

This may be uniquely solved for the connection $\omega_{b}^{a}$. Let

$$
\omega_{b}^{a}=\alpha_{b}^{a}+\beta_{b}^{a},
$$

where $\alpha_{b}^{a}$ is the usual metric compatible spin connection satisfying

$$
\mathbf{d e}^{a}=\mathbf{e}^{b} \wedge \alpha_{b}^{a}
$$

and require

$$
\mathbf{e}^{b} \wedge \beta_{b}^{a}+\omega_{0}^{0} \wedge \mathbf{e}^{a}=0 .
$$

Equation (7.22) is solved uniquely by

$$
\beta_{b}^{a}=-W_{b} \mathbf{e}^{a}+\eta_{b c} \eta^{a d} W_{d} \mathbf{e}^{c},
$$

so we have now satisfied two of the four structure equations.

Next, we impose the tracelessness condition for the space-time term of the curvature in the $\left(\mathbf{e}^{a}, \mathbf{f}_{b}\right)$ basis:

$$
\Omega^{a}{ }_{b a c}=0 .
$$

Rearranging Eq. (2.12a) to solve for $\Omega_{b}^{a}$ we find

$$
\begin{aligned}
\Omega_{b}^{a} & =\mathbf{d} \omega_{b}^{a}-\omega_{b}^{c} \wedge \omega_{c}^{a}-\omega_{b}^{0} \wedge \omega_{0}^{a}+\eta_{b c} \eta^{a d} \omega_{d}^{0} \wedge \omega_{0}^{c} \\
& =\left(\mathbf{d} \alpha_{b}^{a}-\alpha_{b}^{c} \wedge \alpha_{c}^{a}\right)+\left(\mathbf{d} \beta_{b}^{a}-\beta_{b}^{c} \wedge \alpha_{c}^{a}-\alpha_{b}^{c} \wedge \beta_{c}^{a}\right)-\beta_{b}^{c} \wedge \beta_{c}^{a}-\omega_{b}^{0} \wedge \mathbf{e}^{a}+\eta_{b c} \eta^{a d} \omega_{d}^{0} \wedge \mathbf{e}^{c} \\
& \equiv \mathbf{R}_{b}^{a}(\alpha)+\mathbf{D} \beta_{b}^{a}-\beta_{b}^{c} \wedge \beta_{c}^{a}-\omega_{b}^{0} \wedge \mathbf{e}^{a}+\eta_{b c} \eta^{a d} \omega_{d}^{0} \wedge \mathbf{e}^{c},
\end{aligned}
$$

where we have written $\mathbf{D}$ for the covariant exterior derivative using the $\mathbf{e}^{a}$-compatible connection $\alpha_{b}^{a}$, and $\mathbf{R}_{b}^{a} \equiv \mathbf{d} \alpha_{b}^{a}-\alpha_{b}^{c} \wedge \alpha_{c}^{a}$ is the usual Riemann curvature two-form. Substituting Eq. (7.17) for $\omega_{a}^{0}$ and (7.23) for $\beta_{b}^{a}$, some algebra leads to

$$
\Omega_{b}^{a}=\mathbf{R}_{b}^{a}+\eta_{b c}\left[\mathbf{D} W^{c} \mathbf{e}^{a}-\mathbf{D} W^{a} \mathbf{e}^{c}+W^{2} \mathbf{e}^{c} \mathbf{e}^{a}\right]+\mathbf{f}_{b} \mathbf{e}^{a}-\eta_{b c} \eta^{a d} \mathbf{f}_{d} \mathbf{e}^{c}+\mathbf{B}_{b} \mathbf{e}^{a}-\eta_{b c} \eta^{a d} \mathbf{B}_{d} \mathbf{e}^{c} .
$$

Finally, expand

$$
\mathbf{D} W^{a}=-\eta^{a b} \mathbf{D} y_{b}+\mathbf{D} A^{a}
$$

While $\mathbf{D} A^{a}$ is independent of $y_{\mu}$, we must further expand

$$
\mathbf{D} y_{b} \equiv \mathbf{d}\left(e_{b}{ }^{\mu} y_{\mu}\right)-\alpha_{b}^{a} e_{a}{ }^{\mu} y_{\mu}=e_{b}{ }^{\mu} \mathbf{d} y_{\mu}+\left(\mathbf{d} e_{b}{ }^{\mu}-\alpha_{b}^{a} e_{a}{ }^{\mu}\right) y_{\mu} .
$$

The first term in Eq. (7.28) is simply $\mathbf{f}_{a}$. For the final term we use the Christoffel connection $\Gamma^{\mu}{ }_{\alpha \beta}$ and the covariant constancy of $e_{b}{ }^{\mu}$, 


$$
\mathbf{d} x^{\beta} D_{\beta} e_{b}{ }^{\mu}=\mathbf{d} e_{b}{ }^{\mu}-\alpha_{b}^{a} e_{a}{ }^{\mu}+e_{b}{ }^{\alpha} \Gamma^{\mu}{ }_{\alpha \beta} \mathbf{d} x^{\beta}=0
$$

to write

$$
\mathbf{D} y_{b}=\mathbf{f}_{b}-e_{b}^{\alpha} \Gamma_{\alpha \beta}^{\mu} \mathbf{d} x^{\beta} y_{\mu} \equiv \mathbf{f}_{b}-\Gamma_{b c} \mathbf{e}^{c} \equiv \mathbf{f}_{b}-\Gamma_{b} .
$$

Now substitution into Eq. (7.26) shows that the cross and momentum terms of the curvature vanish while the components of the space-time term become

$$
\begin{aligned}
\Omega_{b c d}^{a}= & \mathbf{R}_{b c d}^{a}-\left(D_{c} A_{b}+\beta_{b c}+B_{b c}\right) \delta_{d}^{a}+\left(D_{d} A_{b}+\Gamma_{b d}+B_{b d}\right) \delta_{c}^{a} \\
& +\eta_{b f} \eta^{a g}\left(D_{c} A_{g}+\Gamma_{g c}+B_{g c}\right) \delta_{d}^{f}-\eta_{b f} \eta^{a g}\left(D_{d} A_{g}+\Gamma_{g d}+B_{g d}\right) \delta_{c}^{f} \\
& -W_{c}\left(W_{b} \delta_{d}^{a}-\eta_{b f} \eta^{a g} W_{g} \delta_{d}^{f}\right)+W_{d}\left(W_{b} \delta_{c}^{a}-\eta_{b f} \eta^{a g} W_{g} \delta_{c}^{f}\right)+W^{2}\left(\eta_{b c} \delta_{d}^{a}-\eta_{b d} \delta_{c}^{a}\right)
\end{aligned}
$$

Next, by setting the trace of $\Omega_{b c d}^{a}$ to zero, we can insure that the Einstein equation holds with arbitrary stress-energy tensor. Contracting, we find that the antisymmetric part $\Omega_{a c b}^{c}-\Omega_{b c a}^{c}$ is identically zero while

$$
\Omega_{a c b}^{c}=R_{a b}+2\left(A_{(a ; b)}+\Gamma_{a b}+S_{a b}+W_{a} W_{b}\right)+\eta_{a b}\left(A_{c}{ }^{; c}+\Gamma_{c}{ }^{c}+S_{c}{ }^{c}-2 W^{2}\right)=0
$$

relates $B_{(a b)}=S_{a b}$ to the Ricci tensor and the vector potential. If we require

$$
S_{a b} \equiv \mathscr{T}_{a b}-\left(A_{(a ; b))}+\Gamma_{a b}+W_{a} W_{b}-\frac{1}{2} \eta_{a b} W^{2}\right),
$$

where $\mathscr{T}_{a b} \equiv-\frac{1}{2}\left(T_{a b}-\frac{1}{3} \eta_{a b} T\right)$ and $T_{a b}$ is the electromagnetic stress-energy tensor constructed from $A_{b}$ plus the stress-energy tensor from whatever other phenomenological fields one wishes to add, then Eq. (7.32) reduces to the Einstein equation with source $T_{a b}$. We shall see below that the choice given by Eq. (7.33) for $S_{a b}$ greatly simplifies the expressions for both the curvature and the co-torsion.

Nothing further is required in order to satisfy the final structure equation, Eq. (2.12c). Instead, we define the co-torsion by Eq. $(2.12 \mathrm{c})$, giving

$$
\Omega_{a}^{0}=\mathbf{d} \omega_{a}^{0}-\omega_{a}^{b} \wedge \omega_{b}^{0}-\omega_{a}^{0} \wedge \omega_{0}^{0} .
$$

This is easiest to evaluate if we write $\omega_{a}^{0}$ in terms of $W_{a}$ wherever possible, resulting in

$$
\omega_{a}^{0}=\mathscr{T}_{a}-\mathbf{D} W_{a}-W_{a} \mathbf{W}+\frac{1}{2} W^{2} \eta_{a b} \mathbf{e}^{b} .
$$

Using the tracefree condition (i.e., the Einstein equation) to replace $\mathscr{T}_{a}$ by $\mathscr{R}_{a} \equiv-\frac{1}{2}\left(R_{a b}\right.$ $\left.-\frac{1}{6} \eta_{a b} R\right) \mathbf{e}^{b}$ we find that Eq. (2.12c) takes the form

$$
\Omega_{a}^{0}=\mathbf{D} \omega_{a}^{0}-\beta_{a}^{b} \wedge \omega_{b}^{0}-\omega_{a}^{0} \wedge \mathbf{W}=\mathbf{D} \omega_{a}^{0}+W_{a} \mathbf{d W}-W^{b} \eta_{a c} \mathbf{e}^{c} \omega_{b}^{0}-\omega_{a}^{0} \wedge \mathbf{W} .
$$

Substituting Eq. (7.34) for $\omega_{a}^{0}$ and using the Ricci identity $\mathbf{D}^{2} \omega_{a}=-\mathbf{R}_{a}^{b} \omega_{b}$ (for an arbitrary one-form $\omega_{a}$ ) gives, after several cancellations, the surprisingly simple result

$$
\Omega_{a}^{0}=\mathbf{D} \mathscr{R}_{a}+\left[\mathbf{R}_{a}^{b}-\left(\delta_{a}^{c} \delta_{d}^{b}-\eta^{b c} \eta_{a d}\right) \mathscr{R}_{c} \mathbf{e}^{d}\right] W_{b}=\mathbf{D} \mathscr{R}_{a}+\mathbf{C}_{a}^{b} W_{b},
$$

where $\mathbf{C}_{a}^{b}$ is the Weyl curvature two-form. Notice that all derivatives of the Weyl vector have cancelled, so the resulting co-torsion also has vanishing momentum and cross terms. If we use the contracted second Bianchi identity to write $\mathbf{D} \mathscr{R}_{a}$ as a divergence of the Weyl curvature, we find 


$$
\Omega_{a}^{0}=-D_{b} \mathbf{C}_{a}^{b}+\mathbf{C}_{a}^{b} W_{b},
$$

so the co-torsion is simply the Weyl-covariant (not $\alpha_{b}^{a}$-covariant) divergence of the Weyl curvature tensor, hence a direct measure of the deviation of the underlying space-time from conformally flat. ${ }^{42}$

Finally, remarkable cancellations also occur if we use Eq. (7.33) to replace $\mathbf{B}_{a}$ in the full curvature tensor given by Eq. (7.26). The result is simply

$$
\Omega_{b}^{a}=\mathbf{C}_{a}^{b} .
$$

The biconformal geometry is now fully specified except for the vector potential $A_{\mu}$. This is fixed by the field equation

$$
* \mathbf{d} * \mathbf{d} \omega_{0}^{0}=* \mathbf{d} * \mathbf{d A}=\mathbf{J}
$$

where $\mathbf{J}$ is the electromagnetic current.

To complete the proof of the sufficiency of Eqs. (7.1)-(7.4) for the biconformal space to correspond to an Einstein-Maxwell space-time we only need to show that the biconformal geometry is effectively four-dimensional. This happens because the extra dimensions of the biconformal base space can be identified with the tangent space of the Riemannian space-time. Such an identification works for two reasons. First, the co-vector basis $\mathbf{d} y_{\mu}$ has the same scale and Lorentz transformation properties as the basis vectors $\partial / \partial x^{\mu}$ for the tangent space, so we can identify the bases. Second, since both the co-space and the tangent space may be taken as Minkowski vector spaces (i.e., both are flat), the two complete vector spaces may be identified. Thus, while the coordinates $y_{\mu}$ act as momentumlike components of cotangent vectors $y_{\mu} \mathbf{d} x^{\mu}$, their differentials d $y_{\mu}$ from a tangent basis. As discussed further in the final section, this isomorphism guarantees that the extra four dimensions do not lead to undesired new macroscopic effects.

Notice that, while the content of the biconformal space does not exceed that of an EinsteinMaxwell space-time, we cannot claim that a biconformal space satisfying Eqs. (7.1)-(7.4) is homeomophic to a four-dimensional Weyl geometry because the Weyl vector of the biconformal space retains linear $y_{\mu}$ dependence. It is this difference that leads to the vanishing dilation of the biconformal space.

The converse, that a four-dimensional Einstein-Maxwell space-time extends to a unique biconformal space satisfying Eqs. (7.1)-(7.4), is immediate, since, given the stress-energy tensor and electromagnetic current determining the space-time, we can find the solder form and the electromagnetic vector potential and from these directly write down the connection of the associated biconformal space as

$$
\begin{aligned}
& \omega_{0}^{0}=\left(-y_{\mu}+A_{\mu}\right) \mathbf{d} x^{\mu}, \\
& \omega_{0}^{a}=\mathbf{e}^{a}(x), \\
& \omega_{a}^{0}=\mathscr{T}_{a}-\mathbf{D} W_{a}-W_{a} \mathbf{W}+\frac{1}{2} W^{2} \eta_{a b} \mathbf{e}^{b}, \\
& \omega_{b}^{a}=\alpha_{b}^{a}-W_{b} \mathbf{e}^{a}+\eta_{b c} \eta^{a d} W_{d} \mathbf{e}^{c},
\end{aligned}
$$

where we invert the vector space isomorphism above to map the tangent space into the extra four dimensions of the biconformal space. The form above for the connection leads directly to the biconformal curvatures

$$
\Omega_{0}^{0}=0,
$$




$$
\begin{gathered}
\Omega_{0}^{a}=0, \\
\Omega_{a}^{0}=-D_{b} \mathbf{C}_{a}^{b}+\mathbf{C}_{a}^{b} W_{b}, \\
\Omega_{b}^{a}=\mathbf{C}_{b}^{a},
\end{gathered}
$$

and the field equations are unchanged. In terms of the natural conformal expressions $\mathscr{B}_{a}, \mathscr{T}_{a}$, and $\omega_{0}^{0}$, the field equations are

$$
\begin{gathered}
\mathscr{B}_{a}=\mathscr{T}_{a}, \\
* \mathbf{d} * \mathbf{d} \omega_{0}^{0}=\mathbf{J} .
\end{gathered}
$$

The expressions for the curvature above make use of the tracelessness condition $\Omega_{b a c}^{a}=0$ to write the co-solder form in terms of the Ricci tensor instead of the stress-energy tensor. Considering the form of the curvature if we do not impose the tracelessness condition, we can interpret the Einstein equation as being that condition that reduces $\Omega_{b}^{a}$ and $\Omega_{a}^{0}$ to the Weyl curvature and its divergence, respectively.

It should be pointed out that Eqs. (7.40) are quite remarkable for biconformal curvatures in that none of the curvatures has a cross term or momentum term. Despite the eight-dimensional formulation of the theory, all terms containing $\mathbf{d} y_{\mu}$ have dropped out. The space spanned by the $y$ coordinates is therefore flat, and plays no role in the gravitational effects of this class of models.

At this point, we can only conjecture whether or not these biconformal spaces satisfy some action principle. There are certainly some action densities which are natural candidates. Specifically the curvature-quadratic eight-form $\Omega_{B}^{A} \wedge^{*} \Omega_{B}^{A}$ is closely related to the action of the fourdimensional theories discussed in the Introduction, and clearly has the flat biconformal spaces of Sec. III as particular solutions. However, even expressing the resulting field equations is complicated, first because there are 12 Weyl-invariant parts to the curvature and second because the Bianchi identities used to reduce the field equations are correspondingly lengthy. We therefore postpone further investigation of field actions to subsequent research.

In the final section, we discuss the observability of the extra dimensions of biconformal spaces.

\section{DISCUSSION}

By placing conformal gauging on an eight-dimensional base space instead of the usual fourdimensional base space, we have overcome the long-standing problem of size change in physical models based on scale invariance. In this section, we demonstrate that our interpretation of biconformal gauge theory is consistent with experience. In particular, we examine the observable consequences of the added dimensions with regard to structure and function.

Three techniques have been used for adding extra dimensions in fundamental models of the world: (a) topological compactification of the extra dimensions, (b) construction of laws of motion or field equations that dynamically reduce the extra dimensions to a sufficiently small scale that they play no macroscopic role ${ }^{50}$ or (c) identification of the extra dimensions with everyday properties already associated with macroscopic matter. Technique (a) is routinely employed in Kaluza-Klein field theories, while technique (b) has been used recently ${ }^{51,52}$ in an attempt to associate a fifth dimension with mass. The third technique, (c), was used in the development of special relativity where time came to be seen as a coordinate in a higher- (i.e., four) dimensional space rather than as a parameter of an intrinsically different type on a three-dimensional space. The local interpretation of biconformal space is of type (c). One should therefore not think in terms of compactification or other standard Kaluza-Klein ideas. Instead, we regard the extra four dimensions of the biconformal co-space as familiar, routinely observable macroscopic dimensions, 
and ask whether a coordinate interpretation of four-momentum is consistent with experience. We demonstrate this consistency by focusing on the two necessary axes of correspondence:

Intrinsic structure: The biconformal co-space must have the same mathematical structure and transformation properties as momentum space.

Dynamical function: The biconformal dynamical laws and description of collisions or interactions must accord with experience.

We address each of these in turn, then conclude by citing positive evidence for a coordinate interpretation of momentum.

\section{A. Intrinsic structure}

For either flat or curved space-times, momentum space is the tangent space at each point of space-time. To identify the biconformal co-space with the tangent space we must check that both the Minkowski vector space structure of the tangent space and transformation properties of the tangent basis are reflected in the co-space.

The biconformal co-space is a Minkowski vector space if and only if it is flat. This flatness is obvious for the flat solutions studied in Secs. III-VI, while it is a consequence of the vanishing torsion and dilation in the curved models of Sec. VII. In either of these classes it is therefore possible to set up a vector space isomorphism between the tangent space and the co-space. We note that the same isomorphism also holds between the tangent space of a Riemannian space-time and the co-space coordinates of a normal biconformal space. ${ }^{49}$ Normal spaces are defined to be torsion-free spaces in which the dilational curvature is closed, the Weyl one-form is exact, and the space-time term of the curvature tensor is trace-free. We therefore have two large, disjoint classes of biconformal geometries in which the biconformal co-space is isomorphic to the Riemannian tangent space.

There are two transformation properties of momentum variables which we must also check. First, under Lorentz transformations, $p_{\mu}$ transforms with the inverse to the same transformation as $x^{\mu}$ in flat spaces or $\mathbf{d} x^{\mu}$ in curved spaces. That is, there must not be two independent Lorentz transformations which can be applied to the space and co-space separately. This is a notable property of the eight-dimensional gauging of the four-dimensional conformal group. Because we began with the space-time conformal group, there is only a single set of local Lorentz transformations. Moreover, $y_{\mu}$ has the proper covariant form. The second transformation property required of momentum is that its scaling weight be -1 , since (using Planck's constant) momentum has geometric units of inverse length. This corresponds correctly to the inverse length units of $y_{\mu}$.

\section{B. Dynamical function}

We now consider whether Hamiltonian dynamical laws and our experience of collisions or interactions are consistent with a coordinate interpretation of momentum. There are three essential points concerning a coordinate interpretation which must correspond to our usual experience.

(1) Hamiltonian dynamical equations should describe the classical motion of particles and fields.

(2) While there is no continuity requirement on momenta, coordinates should not change discontinuously.

(3) From our experience we know that collisions only occur between particles which are nearby in their space-time coordinates, and do not necessarily occur when their momentum separation becomes small. We must show how this observation is consistent with the single proper biconformal separation, which depends on both position separation and on momentum separation.

For agreement on point 1, we require a match of dynamical properties between the Hamiltonian dynamics of momentum space and the dynamics of biconformal spaces. In Secs. III-VI, we 
showed that the usual Hamiltonian dynamical picture is a natural property of flat biconformal spaces. Not only do the co-space coordinates act as momenta, but the structure equation

$$
\mathbf{d} \omega_{0}^{0}=\omega_{0}^{a} \omega_{a}^{0}
$$

shows that $\mathbf{d} \omega_{0}^{0}$ is a manifestly closed, nondegenerate two-form, hence symplectic, and we have the usual Hamiltonian dynamical structure associated with one-particle phase space. Indeed, we demonstrate that any Hamiltonian system generates a super-Hamiltonian hypersurface in a unique flat biconformal space, and conversely that a hypersurface in a flat biconformal space gives a unique Hamiltonian system.

In the dilation-free curved geometries of Sec. VII and in normal biconformal spaces we can expect the Hamiltonian dynamical laws of flat biconformal space to hold locally. As long as we look at a sufficiently small neighborhood to permit a single-particle picture, the considerations of Secs. III-VI hold without modification. That fields on space-time also pose no problem may be seen from three different perspectives:

(a) The observable properties of fields are characterized by tensors built on the tangent space, so the isomorphism between the tangent space and the co-space insures that the same properties can be measured in both models.

(b) Fields and their properties are derivable as the continuum limit of many individual particles, so as long as interactions between particles are correctly predicted, the field limit may be expected to hold.

(c) Fields must admit classical, single particle limits. Thus, the highly localized field of an isolated electron moves according to a classical single particle Hamiltonian.

The net effect of these considerations is that there is no particle or field property that one might observe in a flat, dilation-free, or normal biconformal space that could not also be described in the tangent bundle of a four-dimensional Riemannian geometry. Moreover, biconformal spaces automatically predict the presence and form of electromagnetic fields and the Lorentz force law, properties which in a Riemannian space-time must be added by hand.

Now consider point 2 . The nearly instantaneous change of momentum which occurs when, say, a ball bounces off a wall, seems to violate the notion of continuous motion in the momentum dimensions. However, the continuity of the actual dynamics underlying the bounce can be seen in either of two ways. Most simply, we can choose the super-Hamiltonian with a phenomenological potential representing the wall. A realistic potential will not have an actual discontinuity, so the resulting motion predicted by Hamilton's equations [or by the biconformal involution of Eqs. $(4.5 b)$ and $(4.5 \mathrm{c})]$ will be continuous.

A more fundamental way of seeing the continuity of momentum during the collision is to use the electrodynamic law predicted by local biconformal theory to examine the actual motion of each constituent particle of the ball in the appropriate background field. In this view, the apparent discontinuity arises because the region of biconformal space chosen for study is too large for the flat approximation to be valid. Shrinking the region to one where a single-particle interpretation is expected to hold solves the problem.

Moving to point 3, we know that in order to collide or interact strongly, the proper separation of two particles must be small. For this fact to be consistent with our experience that collisions occur whenever the space-time separation becomes small regardless of the momentum separation, is nontrivial. Indeed, recent models ${ }^{51,52}$ in which a fifth dimension proportional to mass is added to space-time fail this test-in those models, two particles of vastly different mass will not generally collide.

In the biconformal models presented here, we find that the proper distance between colliding objects behaves according to our experience. The proper interval is given by the conformal Killing 
metric of Eq. (6.8). While Eq. (6.8) applies to the orthonormal $\left(\omega_{0}^{a}, \omega_{a}^{0}\right)$ basis, we easily change to the $\left(\mathbf{e}^{a}, \mathbf{f}_{a}\right)$ basis, where we find that the metric takes the form

$$
g_{a b}=\left(\begin{array}{cc}
-2 \mathscr{W}_{(a b)} & \delta_{m}{ }^{n} \\
\delta_{n}{ }^{m} & 0
\end{array}\right),
$$

where $\mathscr{W}_{a b}=\mathscr{T}_{a b}+\alpha_{a, b}+W_{a} W_{b}-\frac{1}{2} W^{2} \eta_{a b}$. Therefore the squared interval $d s^{2}$ between the ball and the wall in terms of their phase space separations $\left(\Delta x^{m}, \Delta p_{n}\right)$ is

$$
d s^{2}=-2 \mathscr{W}_{m n} \Delta x^{m} \Delta x^{n}+2 \Delta x^{m} \Delta p_{m}
$$

which vanishes when the spacetime separation $\Delta x^{m}$ vanishes and does not necessarily vanish when the momentum separation $\Delta p_{m}=0$. This is exactly what is required.

In parts A and B we have shown that there is no mystery to the extra four dimensions of the biconformal co-space. Indeed, the coordinates for these extra directions are always immediately available as the energy and momentum of the system under study. Thus, if we want to probe the full biconformal geometry by "walking off" in the extra directions, we already know exactly how to do it. We must vary our energy or the direction of our motion. Any change of the tangent to our world line is a change in location in the momentum sector of biconformal space. Of course, because we are constrained to obey the classical laws of physics, we only experience four degrees of freedom, not eight (except as initial dynamical conditions, when we need all eight). In particular, conservation of energy and momentum constrain motions to $\mathbf{d} y_{\mu}=0$ subspaces.

The new picture would be particularly convincing if there were some direct positive evidence that (perhaps under extreme conditions) the full eight-dimensional character of the world is manifest. For such evidence we must study biconformal spaces in which the fields take on a more general $y$ dependence. Therefore, the following observations are necessarily conjectural, the investigation of the microscopic meaning of biconformal space lying clearly beyond the scope of the present paper. Nonetheless, as long as the deviation from the normal or dilation-free classes are small, we may expect the new evidence to show up as a dependence of some physical fields or parameters on momentum as well as position. We briefly discuss two physical variables which have this property: quantum mechanical wave functions, and running coupling constants.

The most evident dependence of fundamental physics on momentum is the phase space duality found in quantum mechanics. It is well understood that the wave function of a particle may be equally well represented using either momentum or position coordinates. In a very direct sense, the momentum of the particle is used as a coordinate. Representations such as the number basis for the harmonic oscillator, which lies midway between momentum and position variables, are also frequently used.

The fact that we do not independently probe both position and momentum simultaneously suggests that even at the quantum level (at least semi-classically) matter is substantially restricted to the neighborhood of some phase-space hypersurface. This might be understood in terms of biconformal space as near-normal biconformal behavior, with microscopic deviations from an involute or nearly involute subspace. The picture is consistent with the view of the path integral approach, which says that a quantum system essentially probes all phase space paths. In the biconformal picture, a path integral would simply be taken over all eight coordinates.

A second piece of evidence that momentum components act as independent coordinates is the existence of running coupling constants in quantum field theory. It is found experimentally that, at high energies, the strength of the electromagnetic and weak couplings vary with energy. Such energy-momentum dependence is easily understood if energy and momentum are coordinates, but is otherwise a somewhat nontransparent result of detailed field theoretic calculation.

Considerable progress has been made in establishing the connection between quantum systems and scale-invariant systems. ${ }^{53,54}$ It remains to be seen to what degree specific biconformal models can be developed which will make these final observations precise. 
${ }^{1}$ H. Weyl, Sitzung. d. Preuss. Akad. d. Wissensch., 465 (1918); The Principle of Relativity (Dover, New York, 1923), Chap. XI.

${ }^{2}$ H. Weyl, Space-Time-Matter (Dover, New York, 1952), 3rd Ed., Chaps. II and IV, 34+35, p. 242, et seq. Originally: H. Weyl, Raum Zeit Materie (J. Springer, Berlin, 1918).

${ }^{3}$ H. Weyl, Nature (London) 106, 781 (1921); Math. Z. 2, 384 (1918); Ann. Physik 54, 117 (1918); Ann. Physik 59, 101 (1919); Phys. Z. 21, 649 (1920); Ann. Physik 65, 541 (1921); Phys. Z. 22, 473 (1921); Z. Phys. 56, 330 (1929).

${ }^{4}$ W. Pauli, Theory of Relativity, translated by G. Field (Dover, New York, 1958), p. 192.

${ }^{5}$ A. Einstein, S. B. preuss. Akad. Wiss., 478 (1918), including Weyl's reply.

${ }^{6}$ F. London, Z. Phys. 42, 375 (1927).

${ }^{7}$ P. A. M. Dirac, Proc. R. Soc. London, Ser. A 209, 291 (1951).

${ }^{8}$ P. A. M. Dirac, Proc. R. Soc. London, Ser. A 212, 330 (1952).

${ }^{9}$ W. Pauli, Theory of Relativity, translated by G. Field (Dover, New York, 1958), p. 192.

${ }^{10}$ R. Adler, M. Bazin, and M. Schiffer, Introduction to General Relativity (McGraw-Hill, New York, 1965), p. 401.

${ }^{11}$ J. Ehlers, A. E. Pirani, and A. Schild, in General Relativity, edited by L. O'Raifeartaigh (Oxford U.P., Oxford, 1972).

${ }^{12}$ R. Utiyama, Prog. Theor. Phys. 50, 2080 (1973).

${ }^{13}$ P. A. M. Dirac, Proc. R. Soc. London, Ser. A 333, 403 (1973).

${ }^{14}$ P. G. O. Freund, Ann. Physik 84, 440 (1974).

${ }^{15}$ R. Utiyama, Prog. Theor. Phys. 53, 565 (1975).

${ }^{16}$ P. G. Bergmann, Introduction to the Theory of Relativity (Dover, New York, 1976), Chap. XVI.

${ }^{17}$ K. Hayashi, M. Kasuya, and T. Shirafuji, Prog. Theor. Phys. 57, 431 (1977).

${ }^{18}$ K. Hayashi and T. Kugo, Prog. Theor. Phys. 61, 339 (1979).

${ }^{19}$ J. Audretsch, Phys. Rev. D 27, 2872 (1983).

${ }^{20}$ J. Audretsch, F. Gähler, and N. Straumann, Commun. Math. Phys. 95, 41 (1984).

${ }^{21}$ D. Ranganathan, J. Math. Phys. 28, 2437 (1986).

${ }^{22}$ H. Cheng, Phys. Rev. Lett. 61, 2182 (1988).

${ }^{23}$ F. Mansouri, Phys. Rev. Lett. 42, 1021 (1979).

${ }^{24}$ F. Mansouri and C. Schaer, Phys. Lett. B 101, 51 (1981).

${ }^{25}$ A. Ferber and P. G. O. Freund, Nucl. Phys. B 122, 170 (1977).

${ }^{26}$ J. Crispim-Romão, A. Ferber, and P. G. O. Freund, Nucl. Phys. B 126, 429 (1977).

${ }^{27}$ M. Kaku, P. K. Townsend, and P. Van Nieuwenhuizen, Phys. Lett. B 69, 304 (1977).

${ }^{28}$ J. T. Wheeler, Phys. Rev. D 44, 1769 (1991).

${ }^{29}$ É. Cartan, La théorie des groupes finis et continus et la géométrie différentielle (Gauthier-Villars, Paris, 1937); C. W. Misner, K. S. Thorne, and J. A. Wheeler, Gravitation (Freeman, San Francisco, 1970) and refs. therein.

${ }^{30}$ F. Klein, "Erlangerprogram: Vergleichende Betrachtungen über neuere geometrischen Forschungen," (1872), translated in Bull. Am. Math. Soc. 2, 215 (1893).

${ }^{31}$ N. Steenrod, The Topology of Fibre Bundles (Princeton U.P., Princeton, NJ, 1951).

${ }^{32}$ S. Kobayashi and K. Nomizu, Foundations of Differential Geometry (Wiley, New York, 1963).

${ }^{33}$ R. Utiyama, Phys. Rev. 101, 1597 (1956).

${ }^{34}$ T. W. B. Kibble, J. Math. Phys. 2, 212 (1961).

${ }^{35}$ S. W. MacDowell and F. Mansouri, Phys. Rev. Lett. 38, 739 (1977).

${ }^{36}$ P. G. O. Freund, Introduction to Supersymmetry (Cambridge U.P., Cambridge, 1986), Chap. 21, pp. 99-105.

${ }^{37}$ C. Chevalley, Theory of Lie Groups (Princeton U.P., Princeton, NJ, 1946).

${ }^{38} \mathrm{Y}$. H. Clifton, private communication.

${ }^{39}$ T. Eguchi, P. B. Gilkey, and A. J. Hanson, Phys. Rep. 66, 213 (1980).

${ }^{40}$ L. P. Hughston and T. R. Hurd, Phys. Rep. 100(5), 273 (1983).

${ }^{41}$ J. T. Wheeler, Proceedings of the Seventh Marcel Grossman Meeting on General Relativity, edited by R. T. Jantzen and G. M. Keiser (World Scientific, London, 1996), pp. 457-459.

${ }^{42}$ P. Szekeres, Proc. R. Soc. London, Ser. A 274, 206 (1963).

${ }^{43}$ C. W. Misner, K. S. Thorne, and J. A. Wheeler, Gravitation (Freeman, San Francisco, 1973), p. 488.

${ }^{44}$ E. Santamato, Phys. Rev. D 29, 216 (1984).

${ }^{45}$ E. R. Caianiello, M. Gasperini, E. Predazzi, and G. Scarpetta, Phys. Lett. A 132, 2,82 (1988).

${ }^{46}$ J. T. Wheeler, Phys. Rev. D 41, 431 (1990).

${ }^{47}$ E. R. Caianiello, A. Feoli, M. Gasperini, and G. Scarpetta, Int. J. Theor. Phys. 29, 2,131 (1990), and references therein.

${ }^{48}$ J. T. Wheeler, "Why quantum mechanics is complex," to be published in Bull. Astron. Soc. India, Dec. 1997.

${ }^{49}$ J. T. Wheeler, http://xxx.lanl.gov/abs/hep-th/9706215, submitted for publication.

${ }^{50} \mathrm{M}$. Gell-Mann, Cal. Tech. field theory seminar (1985).

${ }^{51}$ B. Mashhoon, H. Liu, and P. Wesson, Phys. Lett. B 331, 305 (1994).

${ }^{52}$ H. Liu and B. Mashhoon, Ann. Phys. 4, 565 (1995).

${ }^{53}$ J. T. Wheeler, "Quanta without quantization," Mod. Phys. Lett. A 12(29), 2175-2181 (1997).

${ }^{54}$ J. T. Wheeler, "String without strings," http://xxx.lanl.gov/abs/hep-th/9706209, submitted for publication. 